\title{
Elliptic problems with convection terms in Orlicz spaces
}

\author{
Giuseppina Barletta \\ Dipartimento di Ingegneria Civile, dell'Energia, dell'Ambiente e dei Materiali \\ Università Mediterranea di Reggio Calabria \\ Via Graziella - Loc. Feo di Vito, 89122, Reggio Calabria (Italy) \\ e-mail: giuseppina.barletta@unirc.it \\ Elisabetta Tornatore \\ Dipartimento di Matematica e Informatica \\ Università di Palermo, \\ Via Archirafi 34, 90123 Palermo (Italy) \\ e-mail: elisa.tornatore@unipa.it
}

\begin{abstract}
The existence of a solution to a Dirichlet problem, for a class of nonlinear elliptic equations, with a convection term, is established. The main novelties of the paper stand on general growth conditions on the gradient variable, and on minimal assumptions on $\Omega$.

The approach is based on the method of sub and supersolutions. The solution is a zero of an auxiliary pseudomonotone operator build via truncation tecniques.

We present also some examples in which we highlight the generality of our growth conditions.
\end{abstract}

\section{Introduction}

We are concerned with Dirichlet problems for elliptic equations of the form

$$
\begin{cases}-\operatorname{div}\left(A^{\prime}(|\nabla u|) \frac{\nabla u}{|\nabla u|}\right)=f(x, u, \nabla u) & \text { in } \Omega \\ u=0 & \text { on } \partial \Omega\end{cases}
$$

where $\Omega$ is an open set in $\mathbb{R}^{n}, n \geq 2$, with finite Lebesgue measure $|\Omega|, f: \Omega \times \mathbb{R} \times \mathbb{R}^{n} \rightarrow \mathbb{R}$ is a Carathéodory function and $A:[0, \infty) \rightarrow[0, \infty)$ is a continuously differentiable, strictly convex function, vanishing at 0 , and such that $A^{\prime}(0)=0$.

We emphasize that the convection term $f(x, u, \nabla u)$ depends not only on the solution $u$, but also on its gradient $\nabla u$ and this makes variational methods not applicable. The interest of the problem is witnessed by several contributions on existence results for Dirichlet problems with the convection

Mathematics Subject Classification: 35J25, 35J99, 46 E35.

Keywords: Nonlinear elliptic equations; Orlicz-Sobolev spaces; gradient dependence; subsolution and supersolution, truncation methods.

The authors are members of the Gruppo Nazionale per l'Analisi Matematica, la Probabilità e le loro Applicazioni (GNAMPA) of the Istituto Nazionale di Alta Matematica (INdAM). This research is partially supported by the Ministry of Education, University and Research of Italy, Prin 2017 Nonlinear Differential Problems via Variational, Topological and Set-valued Methods (Project No. 2017AYM8XW). 
term and the $p$-Laplace or the $(p, q)$-Laplace operator ([FGM, FMMT, FMP, G, MMM, MT, MW, $\mathrm{R}]$ ), and also for more general operators ([DF, GW, T]).

We prove some existence results for (1.1) under general assumptions an $A$. Our abstract framework is that of Orlicz spaces and this allows $A$ to be a Young function, not necessarily of power type. This also implies a choice of functions $f$ wider than allowed in [FGM, FMMT, FMP, G, MMM, MT, MW, R]. The growth of $f$ is now related (in a way that we will explain better later) to the function $A$.

Our approach to (1.1) rests upon the use of sub and supersolutions (see Section 3 for the definitions). Related techniques can be found in [DF, FMMT, FMP, G, MMM, MT]. An abstract existence result for (1.1) can be proved, via the theorem on pseudomonotone operators (Theorem 4.9), provided that a subsolution and a supersolution exist. Various results in the literature require additional assumptions on $f$, used only to build a subsolution and a supersolution (see [FMMT, FMP, MMM, $\mathrm{MT}]$ ). Other authors are not just concerned with the existence of subsolutions and supersolutions (see [DF, G]). By contrast, two of our results are formulated under a weaker unified assumption on $f$, which guarantees the existence of a subsolution and a supersolution and enable us to apply a new abstract existence result (Theorem 3.6) for problem (1.1), in which the existence of a subsolution and a supersolution is required.

As far as the regularity of $\Omega$ is concerned, we note that the results mentioned above hold in bounded sets with a sufficiently smooth boundary. We are able to substantially weaken these assumptions. For instance, $\Omega$ can be an open set having finite measure and satisfying the cone property. In fact, we can allow for arbitrary open sets $\Omega$ with finite measure, under additional suitable a priori regularity properties of sub and the supersolutions.

Some more detailed comments on the growth condition on the gradient variable. Unlike most papers dealing with this kind of problem ([FMMT, FMP, G, MMM, MT]), the growth condition in the gradient variable on $A$ and $f$ are not necessarily of power type. In particular $|f|$ is assumed to be bounded from above respect to the gradient in terms of a Young function $E$ that growths essentially more slowly then the optimal Sobolev conjugate of $A$. Our hypotheses on the function $A$ embrace the $p$-Laplacian, the $(p, q)$-Laplacian, the $p$ mean curvature operator $(p>1)$, and functions of the form $A(t)=t^{p} \lg ^{\alpha}(c+t)$, for suitable $\alpha$ and $c$. Related results in this direction can be found in $[\mathrm{DF}, \mathrm{GW}$, where the general setting is that of Musielak-Orlicz-Sobolev spaces. In [DF] a more restrictive definition of sub and supersolution is adopted and prevents from using constant sub or supersolutions. In $[\mathrm{GW}]$ the authors study a double phase problem with a convection term via the theorem on pseudomonotone operators.

The paper is arranged as follows. In Section 2 we introduce the Orlicz-Sobolev spaces that we use throughout the paper. In Section 3 we state our main Theorems and present some applications of Theorem 3.6, in which we highlight how the function $f$ can have a behavior that is outside the classical settings. In Section 4 we prove the technical auxiliary results that we use in Section 5. In particular, we present some new properties of the truncation and of the Nemytski operator in the new setting. All these results are known in the classical situation but are new in our framework, where a $\Delta_{2}$ condition plays a crucial role. The main results are proved in Section 5 .

\section{Young functions and Orlicz spaces}

In this Section we give the main definitions on Young functions, introduce the Orlicz Sobolev spaces that we use throughout the paper and present some of their basic properties. For a comprehensive treatment of Young functions and Orlicz spaces we refer the reader to the monographs [HH, KrRu, RR1, RR2]. At the end of the Section we present our main results. 
Definition 2.1 A function $A:[0, \infty) \rightarrow[0, \infty]$ is called a Young function if it is convex, vanishes at 0 , and is neither identically equal to 0 , nor to infinity.

For any a Young function $A$, one has

$$
A(\lambda t) \geq \lambda A(t) \text { for all } \lambda \geq 1, t \geq 0 .
$$

Definition 2.2 A Young function $A$ is said to dominate another Young function $B$ near infinity (briefly $B \leq A$ near infinity), if there exist constants $c_{0}>0$ and $M \geq 0$ such that

$$
B(t) \leq A\left(c_{0} t\right) \text { for all } t \geq M
$$

If (2.1) holds with $M=0$, then we say that $A$ dominates $B$ globally. Two Young functions $A$ and $B$ are called equivalent near infinity [globally] if they dominate each other near infinity [globally].

Definition 2.3 The function $B$ is said to increase essentially more slowly than $A$ near infinity (briefly $B \ll A$ ), if

$$
\lim _{t \rightarrow \infty} \frac{B(\lambda t)}{A(t)}=0 \text { for all } \lambda>0
$$

Condition (2.2) is equivalent to

$$
\lim _{s \rightarrow \infty} \frac{A^{-1}(s)}{B^{-1}(s)}=0
$$

Here $A^{-1}$ and $B^{-1}$ are the (generalized) left-continuous inverse of $A$ and of $B$ respectively.

Note that if $A$ dominates $B$ near infinity but they are not equivalent near infinity then $B \ll A$.

Definition 2.4 The Young conjugate of a Young function $A$ is the Young function $\widetilde{A}$ defined as

$$
\widetilde{A}(s)=\sup \{s t-A(t): t \geq 0\} \quad \text { for } s \geq 0 .
$$

For any Young function $A$ one has $\widetilde{\widetilde{A}}=A$,

$$
t s \leq A(t)+\widetilde{A}(s) \text { for } t, s \geq 0
$$

and

$$
t \leq \widetilde{A}^{-1}(t) A^{-1}(t) \leq 2 t \text { for } t \geq 0 .
$$

Hence,

$$
\frac{A(t)}{t} \leq \widetilde{A}^{-1}(A(t)) \leq 2 \frac{A(t)}{t} \text { for } t>0
$$

Further, if $B$ is finite valued and $A \ll B$ then $\widetilde{B} \ll \widetilde{A}$ (see [BC], Proposition A4).

Definition 2.5 A Young function $A$ is said to satisfy the $\Delta_{2}$-condition near infinity (briefly $A \in \Delta_{2}$ near infinity) if it is finite valued and there exist constants $K \geq 2$ and $M \geq 0$ such that

$$
A(2 t) \leq K A(t) \quad \text { for } t \geq M \text {. }
$$


Definition 2.6 The function $A$ is said to satisfy the $\nabla_{2}$-condition near infinity (briefly $A \in \nabla_{2}$ near infinity) if there exist constants $K>2$ and $M \geq 0$ such that

$$
A(2 t) \geq K A(t) \quad \text { for } t \geq M \text {. }
$$

If (2.6) or (2.7) holds with $M=0$, then $A$ is said to satisfy the $\Delta_{2}$-condition (globally), or the $\nabla_{2^{-}}$ condition (globally), respectively. If $A$ and $B$ are equivalent near infinity then $A \in \Delta_{2}$ near infinity if and only if $B \in \Delta_{2}$ near infinity.

Let us now introduce the Orlicz spaces where we work together with their main properties. We state them in the general setting, and we specify from time to time if some additional condition is needed for them to hold. Let $\Omega$ be a measurable set in $\mathbb{R}^{n}, n \geq 1$. Given a Young function $A$, the Orlicz space $L^{A}(\Omega)$ is the set of all measurable functions $u: \Omega \rightarrow \mathbb{R}$ such that the Luxemburg norm

$$
\|u\|_{L^{A}(\Omega)}=\inf \left\{\lambda>0: \int_{\Omega} A\left(\frac{1}{\lambda}|u|\right) d x \leq 1\right\}
$$

is finite. The functional $\|\cdot\|_{L^{A}(\Omega)}$ is a norm on $L^{A}(\Omega)$, and it is a Banach space (see [Ad]). If $A$ is a Young function and $\widetilde{A}$ denotes its conjugate, then a generalized Hölder inequality

$$
\int_{\Omega}|u v| d x \leq 2\|u\|_{L^{A}(\Omega)}\|v\|_{L^{\widetilde{A}}(\Omega)}
$$

holds for every $u \in L^{A}(\Omega)$ and $v \in L^{\widetilde{A}}(\Omega)$.

From [RR1, Theorem 12, Chapter 3] we know that for $v \in L^{A}(\Omega)$ and $\left\{v_{k}\right\} \subset L^{A}(\Omega)$ one has

$$
\lim _{k \rightarrow \infty}\left\|v_{k}-v\right\|_{L^{A}(\Omega)}=0
$$

if and only if

$$
\lim _{k \rightarrow \infty} \int_{\Omega} A\left(\frac{\left|v_{k}-v\right|}{\lambda}\right) d x=0 \text { for every } \lambda>0 .
$$

Further, if (2.10) is in force, then up to a subsequence, we can find $w \in L^{A}(\Omega)$ such that $\left|v_{k}(x)\right| \leq$ $w(x)$ a.e. in $\Omega$ for all $k \in \mathbb{N}$. The proof of this fact follows the same lines of that of the classical situation where $L^{A}(\Omega)=L^{p}(\Omega)$.

Finally, if (2.6) holds, then

$$
\int_{\Omega} A(\lambda|u|) d x<+\infty \quad \text { for every } \lambda>0, \quad \text { for every } u \in L^{A}(\Omega) .
$$

This fact is no longer true when (2.6) fails.

Let $A$ and $B$ be Young functions such that $A$ dominates $B$ near infinity. If $|\Omega|<\infty$, we have the embedding

$$
L^{A}(\Omega) \rightarrow L^{B}(\Omega)
$$

In particular,

$$
L^{A}(\Omega) \rightarrow L^{1}(\Omega)
$$

for any Young function $A$. Moreover

$$
L^{A}(\Omega) \text { is reflexive if and only if } A \in \Delta_{2} \cap \nabla_{2} \text { near infinity }
$$


(see [Sch, Corollary 7.2]).

Given any Young function $A \in C^{1}([0,+\infty))$, define the quantities

$$
i_{A}=\liminf _{t \rightarrow+\infty} \frac{t \cdot A^{\prime}(t)}{A(t)}, \quad s_{A}=\limsup _{t \rightarrow+\infty} \frac{t \cdot A^{\prime}(t)}{A(t)} .
$$

One has $1 \leq i_{A} \leq s_{A} \leq+\infty$. The conditions

$$
i_{A}>1 \text { and } s_{A}<+\infty
$$

are equivalent to $A \in \nabla_{2} \cap \Delta_{2}$ near infinity. Finally, we introduce another quantity that comes into play in our main result:

$$
p_{A}=\inf _{t>0} \frac{t \cdot A^{\prime}(t)}{A(t)} .
$$

The condition $p_{A}>1$ is equivalent to $A \in \nabla_{2}$ globally. Also, if $p_{A}>1$ then $A^{\prime}(0)=0$, and (see [CM, Lemma A.1]

$$
\int_{\Omega} A(|v|) d x \geq\|v\|_{L^{A}(\Omega)}^{p_{A}} \text { for every } v \in L^{A}(\Omega), \text { such that }\|v\|_{L^{A}(\Omega)} \geq 1 .
$$

In the main results we require

$$
p_{A}>1 \text { and } s_{A}<+\infty \text {. }
$$

The first inequality is crucial in the proof of the coercivity of a suitable functional, the other one in many technical lemmas.

The Lemma below extends (2.11). It will be used several times in the paper, either to prove the well posedness of some functionals or the summability of suitable functions.

Lemma 2.7 Assume that $|\Omega|<\infty$. Let $C$ and $D$ be two Young functions such that $D \ll C$ near infinity or $D$ and $C$ are equivalent near infinity and $D \in \Delta_{2}$ near infinity. Then for every $\lambda, k>0$ there exists a constant $c=c(\lambda, k, D, C,|\Omega|)$ such that

$$
\sup _{\|u\|_{L} C(\Omega)} \leq k \int_{\Omega} D(\lambda|u|) d x \leq c,
$$

and

$$
\sup _{\|u\|_{L^{C}(\Omega)} \leq k}\left\|G^{-1}(D(\lambda|u|))\right\|_{L^{G}(\Omega)} \leq \max \{1, c\} \quad \text { for every Young function } G \text {. }
$$

Proof. In the proof $\|u\|_{C}$ stands for $\|u\|_{L^{C}(\Omega)}$. Let us consider first the case in which $D \ll C$ near infinity. Given $\lambda, k>0$ there exists $t_{0}>0$ such that

$$
D(\lambda k t) \leq C(t) \quad \text { if } \quad t \geq t_{0}
$$

Thus, if $\|u\|_{C} \leq k$, then

$$
\int_{\Omega} D(\lambda|u|) d x=\int_{\left\{\frac{|u|}{\|u\|_{C}} \leq t_{0}\right\}} D\left(\lambda k \frac{|u|}{\|u\|_{C}}\right) d x+\int_{\left\{\frac{|u|}{\|u\|_{C}}>t_{0}\right\}} C\left(\frac{|u|}{\|u\|_{C}}\right) d x \leq D\left(\lambda k t_{0}\right)|\Omega|+1 .
$$


Choose $c=D\left(\lambda k t_{0}\right)|\Omega|+1$ and (2.17) follows.

(2.18) can be deduced from the inequalities below, via the very definition of the Luxemburg norm.

$$
\int_{\Omega} G\left(\frac{\left(G^{-1}\right) D(\lambda|u|)}{\max \{1, c\}}\right)<\frac{1}{\max \{1, c\}} \int_{\Omega} D(\lambda|u|) d x \leq 1 .
$$

Assume now that $D$ and $C$ are equivalent near infinity and $D \in \Delta_{2}$ near infinity. Let $M$ and $K$ be as in (2.6). Given $\lambda, k>0$, choose $h$ satisfying $\lambda k \leq 2^{h}$. Then

$$
\begin{aligned}
& \int_{\Omega} D(\lambda|u|) \leq \int_{\Omega} D\left(\lambda k \frac{|u|}{\|u\|_{C}}\right) d x \leq \int_{\left\{\frac{|u|}{\|u\|_{C}} \leq M\right\}} D(\lambda k M) d x \\
&+\int_{\left\{\frac{|u|}{\|u\|_{C}}>M\right\}} K^{h} D\left(\frac{|u|}{\|u\|_{C}}\right) d x \leq D(\lambda k M)|\Omega|+K^{h} .
\end{aligned}
$$

We can now argue as for previous case.

From now on, we assume that $\Omega$ is an open set in $\mathbb{R}^{n}$ and $|\Omega|<\infty$. The isotropic Orlicz-Sobolev spaces $W^{1, A}(\Omega)$ and $W_{0}^{1, A}(\Omega)$ are defined as

$$
W^{1, A}(\Omega)=\left\{u: \Omega \rightarrow \mathbb{R}: u \text { is weakly differentiable in } \Omega, \quad u,|\nabla u| \in L^{A}(\Omega)\right\}
$$

and

$$
\begin{aligned}
W_{0}^{1, A}(\Omega)=\{u: \Omega \rightarrow \mathbb{R}: & \text { the continuation of } u \text { by } 0 \text { outside } \Omega \\
& \text { is weakly differentiable in } \left.\mathbb{R}^{n}, \quad u,|\nabla u| \in L^{A}(\Omega)\right\} .
\end{aligned}
$$

The space $W^{1, A}(\Omega)$ equipped with the norm

$$
\|u\|_{W^{1, A}(\Omega)}=\|u\|_{L^{A}(\Omega)}+\|\nabla u\|_{L^{A}(\Omega)}
$$

is a Banach space. Also, on the Banach space $W_{0}^{1, A}(\Omega)$ we can use the equivalent norm

$$
\|u\|_{W_{0}^{1, A}(\Omega)}=\|\nabla u\|_{L^{A}(\Omega)} .
$$

From (2.13) we know that

$$
W^{1, A}(\Omega) \rightarrow W^{1,1}(\Omega) \quad \text { for every Young function } A .
$$

If $A \in \Delta_{2} \cap \nabla_{2}$ near infinity, then the Orlicz-Sobolev space $W_{0}^{1, A}(\Omega)$ is reflexive ([BC], Proposition $3.1)$.

Definition 2.8 (see [Ci3]) The optimal Sobolev conjugate of $A$ is defined by $A_{n}:[0, \infty) \rightarrow[0, \infty]$

$$
A_{n}(t)=A\left(H^{-1}(t)\right) \quad \text { for } t \geq 0,
$$

where $H:[0, \infty) \rightarrow[0, \infty)$ is given by

$$
H(t)=\left(\int_{0}^{t}\left(\frac{\tau}{A(\tau)}\right)^{\frac{1}{n-1}} d \tau\right)^{\frac{n-1}{n}} \quad \text { for } t \geq 0
$$

provided that the integral is convergent. Here, $H^{-1}$ denotes the generalized left-continuous inverse of $H$. 
Note that if $A \ll B$ then $A_{n} \ll B_{n}$ too.

Before stating the optimal embedding theorem for $W^{1, A}(\Omega)$ we set

$$
\begin{array}{r}
\mathcal{G}\left(\frac{1}{n^{\prime}}\right)=\left\{G \subset \mathbb{R}^{n}: G \text { is open and there exist two positive constants } Q, N\right. \\
\text { such that } \left.|E|^{\frac{1}{n^{\prime}}} \leq Q P(E ; G) \text { for all } E \subset G \text { with }|E| \leq N\right\},
\end{array}
$$

here $P(E ; G)$ is the perimeter of $E$ relative to $G$ (see $[\mathrm{M}])$ and $n^{\prime}=\frac{n}{n-1}$.

Any bounded set $G$ having the cone property belongs in $\mathcal{G}\left(\frac{1}{n^{\prime}}\right)$.

Let now $\Omega \in \mathcal{G}\left(\frac{1}{n^{\prime}}\right)$. Assume that $\Omega$ has finite measure and

$$
\int_{0}\left(\frac{\tau}{A(\tau)}\right)^{\frac{1}{n-1}} d \tau<\infty
$$

then (see [Ci1, Theorem 2]

$$
W^{1, A}(\Omega) \rightarrow L^{A_{n}}(\Omega) .
$$

Thus from [Ci1, Theorem 3] (or [LI, Theorem 3.1]), the embedding $W^{1, A}(\Omega) \rightarrow L^{E}(\Omega)$ is compact for every Young function $E \ll A_{n}$ near infinity.

If $\Omega$ is an open subset in $\mathbb{R}^{n}$ having finite measure then (see [Ci3, Theorem 1])

$$
W_{0}^{1, A}(\Omega) \rightarrow L^{A_{n}}(\Omega),
$$

and there exists a constant $C=C(n)$ such that

$$
\|u\|_{L^{A_{n}(\Omega)}} \leq C\|u\|_{W_{0}^{1, A}(\Omega)} \text { for every } u \in W_{0}^{1, A}(\Omega) .
$$

Moreover, the embedding $W_{0}^{1, A}(\Omega) \rightarrow L^{E}(\Omega)$ is compact for every Young function $E \ll A_{n}$ near infinity.

If

$$
\int^{\infty}\left(\frac{\tau}{A(\tau)}\right)^{\frac{1}{n-1}} d \tau=\infty
$$

then $A_{n}$ assumes only finite values, while when

$$
\int^{\infty}\left(\frac{\tau}{A(\tau)}\right)^{\frac{1}{n-1}} d \tau<\infty
$$

then $A_{n}(t)=\infty$ for $t$ large and (2.26) yields

$$
\|u\|_{L^{\infty}(\Omega)} \leq C\|u\|_{W_{0}^{1, A}(\Omega)}
$$

for every $u \in W_{0}^{1, A}(\Omega)$.

When (2.27) holds then (see [BC, (3.13)])

$$
\int_{\Omega} A_{n}(\lambda|u|) d x<\infty \text { for every } u \in W_{0}^{1, A}(\Omega), \text { and every } \lambda>0 .
$$

It is worthwhile to point a partial extension of (2.24), (2.25) and (2.26) when (2.23) fails. If (2.23) fails then we can substitute $A$ with another function $B$ equivalent to $A$ near infinity and such that (2.23) holds (in particular $B>A$ near 0). Due to $|\Omega|<\infty, W_{0}^{1, A}(\Omega)=W_{0}^{1, B}(\Omega)$ and (2.24), (2.25) and (2.26) hold with $B_{n}$. In the future, according to this convention, we denote always with $A_{n}$ the optimal conjugate in (2.26).

The Proposition below establishes a new property for the function $A_{n}$. 
Proposition 2.9 Let $A \in C^{1}([0, \infty))$ be a Young function and let $A_{n}$ be its Sobolev conjugate, defined as in (2.22). Then

$$
\liminf _{t \rightarrow+\infty} \frac{t \cdot A_{n}^{\prime}(t)}{A_{n}(t)} \geq \frac{n}{n-1}
$$

and $A_{n} \in \nabla_{2}$ at infinity.

Proof. We consider only the case in which (2.27) holds. The function $H$ is increasing and $H \in$ $C^{1}([0,+\infty))$. Making the change of variable $H^{-1}(t)=s$, and using the inequality $A^{\prime}(s)>\frac{A(s)}{s} \forall s>$ 0

$$
\begin{gathered}
\frac{t \cdot A_{n}^{\prime}(t)}{A_{n}(t)}=\frac{t \cdot \frac{A^{\prime}\left(H^{-1}(t)\right)}{H^{\prime}(s)_{\mid s=H^{-1}(t)}}}{A\left(H^{-1}(t)\right)}=\frac{H(s) A^{\prime}(s)}{H^{\prime}(s) A(s)}=\frac{\left(\int_{0}^{s}\left(\frac{\tau}{A(\tau)}\right)^{\frac{1}{n-1}} d \tau\right)^{\frac{n-1}{n}} \cdot A^{\prime}(s)}{\frac{n-1}{n}\left(\int_{0}^{s}\left(\frac{\tau}{A(\tau)}\right)^{\frac{1}{n-1}} d \tau\right)^{-\frac{1}{n}} \cdot\left(\frac{s}{A(s)}\right)^{\frac{1}{n-1}} \cdot A(s)} \\
=\frac{n}{n-1} \cdot \frac{\left(\int_{0}^{s}\left(\frac{\tau}{A(\tau)}\right)^{\frac{1}{n-1}} d \tau\right) \cdot A^{\prime}(s) \cdot(A(s))^{\frac{2-n}{n-1}}}{s^{\frac{1}{n-1}} \frac{n}{n-1} \cdot \frac{\left(\int_{0}^{s}\left(\frac{\tau}{A(\tau)}\right)^{\frac{1}{n-1}} d \tau\right) \cdot \frac{A(s)}{s} \cdot(A(s))^{\frac{2-n}{n-1}}}{s^{\frac{1}{n-1}}}} \\
=\frac{n}{n-1} \cdot \frac{\left(\int_{0}^{s}\left(\frac{\tau}{A(\tau)}\right)^{\frac{1}{n-1}} d \tau\right) \cdot(A(s))^{\frac{1}{n-1}}}{s^{\frac{n}{n-1}}} \\
\text { for } t>0, s=H^{-1}(t) .
\end{gathered}
$$

Thus

$$
\liminf _{t \rightarrow+\infty} \frac{t \cdot A_{n}^{\prime}(t)}{A_{n}(t)} \geq \liminf _{s \rightarrow+\infty} \frac{n}{n-1} \cdot \frac{\left(\int_{0}^{s}\left(\frac{\tau}{A(\tau)}\right)^{\frac{1}{n-1}} d \tau\right) \cdot(A(s))^{\frac{1}{n-1}}}{s^{\frac{n}{n-1}}} .
$$

Let

$$
l=\liminf _{s \rightarrow+\infty} \frac{n}{n-1} \cdot \frac{\left(\int_{0}^{s}\left(\frac{\tau}{A(\tau)}\right)^{\frac{1}{n-1}} d \tau\right) \cdot(A(s))^{\frac{1}{n-1}}}{s^{\frac{n}{n-1}}} .
$$

If $l=+\infty$ then we finished. Otherwise, using the De L'Hopital theorem (a version with the $\liminf s \rightarrow+\infty \frac{f(s)}{g(s)}$ for positive $f$ and $g$ )

$$
\begin{aligned}
l \geq \liminf _{s \rightarrow+\infty} \frac{\frac{n}{n-1} \cdot\left[\left(\frac{s}{A(s)}\right)^{\frac{1}{n-1}} \cdot(A(s))^{\frac{1}{n-1}}+\frac{1}{n-1}\left(\int_{0}^{s}\left(\frac{\tau}{A(\tau)}\right)^{\frac{1}{n-1}} d \tau\right) \cdot(A(s))^{\frac{1}{n-1}-1} \cdot A^{\prime}(s)\right]}{\frac{n}{n-1} s^{\frac{1}{n-1}}} \\
\quad \geq \liminf _{s \rightarrow+\infty} \frac{\left[s^{\frac{1}{n-1}}+\frac{1}{n-1}\left(\int_{0}^{s}\left(\frac{\tau}{A(\tau)}\right)^{\frac{1}{n-1}} d \tau\right) \cdot(A(s))^{\frac{1}{n-1}-1} \cdot \frac{A(s)}{s}\right]}{s^{\frac{1}{n-1}}}=1+\frac{l}{n} .
\end{aligned}
$$

From (2.32) we deduce $l \geq \frac{n}{n-1}$, and (2.30) follows. Since $\frac{n}{n-1}>1$ we conclude that $A_{n} \in \nabla_{2}$ at infinity. 


\section{Main Results and Examples}

In this Section we state the main results of the paper (Theorems 3.2, 3.3 and 3.6) and present some examples.

First we give the fundamental definition of weak solution to (1.1).

Definition 3.1 We say that $u \in W_{0}^{1, A}(\Omega)$ is a weak solution to problem (1.1) if

$$
\int_{\Omega} A^{\prime}(|\nabla u|) \frac{\nabla u}{|\nabla u|} \nabla v d x=\int_{\Omega} f(x, u, \nabla u) v d x
$$

for all $v \in W_{0}^{1, A}(\Omega)$, and $f(x, u, \nabla u) v \in L^{1}(\Omega)$ for every $u, v \in W_{0}^{1, A}(\Omega)$.

We introduce some functions that come into play in Theorems 3.2 and 3.3. Even if there is a difference between the behaviour allowed for $f$ in the first and in the second result, the assumptions on the functions involved are the same, so for simplicity we list them here.

We assume

$$
(\mathcal{H}):\left\{\begin{array}{c}
E:\left[0,+\infty\left[\rightarrow \left[0,+\infty\left[\text { is a Young function, } E \ll A_{n} \text { near infinity and } a \geq 0\right. \text { is a constant, }\right.\right.\right. \\
\rho_{1}, \rho_{2}: \Omega \rightarrow\left[0,+\infty\left[\text { are two measurable functions, } \rho_{i} \in L^{\widetilde{A}_{n}}(\Omega), i=1,2,\right. \text { and }\right. \\
\quad \rho_{2}(x)>0 \text { on a set of positive measure; } \\
g_{1}, g_{2}:\left[0,+\infty\left[\rightarrow \left[0,+\infty\left[\text { are two non decreasing functions such that } g_{1}(0)=g_{2}(0)=0\right.\right.\right.\right. \\
\text { and there exist } \left.s_{0}>0, k_{1} \in\right] 0, \omega_{n}^{\frac{1}{n}}|\Omega|^{-\frac{1}{n}}\left[, k_{2}>0\right. \text { such that } \\
g_{1}(|s|)|s| \leq A\left(k_{1}|s|\right) \text { and } g_{2}(|s|)|s| \leq A_{n}\left(k_{2}|s|\right) \text { for all }|s| \geq s_{0} .
\end{array}\right.
$$

Here $\omega_{n}$ is the measure of the unit ball in $\mathbb{R}^{n}$.

Theorem 3.2 Let $\Omega$ be an open set in $\mathbb{R}^{n}$, with $n \geq 2$, such that $|\Omega|<\infty$. Let $A \in C^{1}([0,+\infty))$ be a Young function for which (2.16) holds. Assume also that A satisfies (2.23) and (2.27), or (2.28). Let $f: \Omega \times \mathbb{R} \times \mathbb{R}^{n} \rightarrow \mathbb{R}$ be a Carathéodory function fulfilling

$$
-\rho_{1}(x)-g_{1}(|s|) \leq f(x, s, \xi) \leq-\rho_{2}(x)+g_{2}(|s|)+a \widetilde{E}^{-1}(A(|\xi|)) \text { for a.e. } x \in \Omega \text {, all }(s, \xi) \in \mathbb{R} \times \mathbb{R}^{n},
$$

where a, $E, \rho_{1}, \rho_{2}, g_{1}, g_{2}$ are as in $(\mathcal{H})$. Then problem $(P)$ possesses a nontrivial solution $u \in$ $W_{0}^{1, A}(\Omega)$ such that $u \leq 0$ a.e. in $\Omega$.

Theorem 3.3 Let $\Omega$ be an open set in $\mathbb{R}^{n}$, with $n \geq 2$, such that $|\Omega|<\infty$. Let $A \in C^{1}([0,+\infty))$ be a Young function for which (2.16) holds. Assume also that A satisfies (2.23) and (2.27), or (2.28). Let $f: \Omega \times \mathbb{R} \times \mathbb{R}^{n} \rightarrow \mathbb{R}$ be a Carathéodory function fulfilling

$$
\rho_{2}(x)-g_{2}(|s|)-a \widetilde{E}^{-1}(A(|\xi|)) \leq f(x, s, \xi) \leq \rho_{1}(x)+g_{1}(|s|) \quad \text { for a.e. } x \in \Omega \text {, all }(s, \xi) \in \mathbb{R} \times \mathbb{R}^{n} \text {, }
$$

where a, $E, \rho_{1}, \rho_{2}, g_{1}, g_{2}$ are as in $(\mathcal{H})$. Then problem $(P)$ possesses a nontrivial solution $u \in$ $W_{0}^{1, A}(\Omega)$ such that $u \geq 0$ a.e. in $\Omega$.

To prove the Theorems above we need a new abstract existence result for problem (1.1), Theorem 3.6 below. Before stating it, we introduce the new definitions of sub and supersolution. These definitions are adapted to the new contest, but they coincide with the classic ones for smooth domains.

Definition 3.4 We say that $\bar{u} \in W^{1, A}(\Omega)$ is a supersolution to $(1.1)$ if $(\bar{u})^{-} \in W_{0}^{1, A}(\Omega)$ and

$$
\int_{\Omega} A^{\prime}(|\nabla \bar{u}|) \frac{\nabla \bar{u}}{|\nabla \bar{u}|} \nabla v d x \geq \int_{\Omega} f(x, \bar{u}, \nabla \bar{u}) v d x
$$

for all $v \in W_{0}^{1, A}(\Omega), v \geq 0$ a.e. in $\Omega$. 
Definition 3.5 We say that $\underline{u} \in W^{1, A}(\Omega)$ is a subsolution to $(1.1)$ if $\underline{u}^{+} \in W_{0}^{1, A}(\Omega)$ and

$$
\int_{\Omega} A^{\prime}(|\nabla \underline{u}|) \frac{\nabla \underline{u}}{|\nabla \underline{u}|} \nabla v d x \leq \int_{\Omega} f(x, \underline{u}, \nabla \underline{u}) v d x
$$

for all $v \in W_{0}^{1, A}(\Omega), v \geq 0$ a.e. in $\Omega$.

Any subsolution and any supersolution to problem (1.1) belong in $W^{1, A}(\Omega)$ and thus in $L^{A}(\Omega)$. In the following Theorem we require a slightly stronger property: $\underline{u}, \bar{u} \in L^{A_{n}}(\Omega)$. This condition is verified, for instance, when $\Omega \in \mathcal{G}\left(\frac{1}{n^{\prime}}\right)$. We note also that, due to $|\Omega|<\infty$, all essentially bounded sub and supersolutions belong in $L^{A_{n}}(\Omega)$. The same holds for sub and supersolutions belonging in $W_{0}^{1, A}(\Omega)$.

Theorem 3.6 Let $\Omega$ be an open set in $\mathbb{R}^{n}$, with $n \geq 2$, such that $|\Omega|<\infty$. Let $A \in C^{1}([0,+\infty))$ be a Young function for which (2.16) holds. Assume also that A satisfies (2.23) and (2.27), or (2.28). Let $\underline{u}$ and $\bar{u}$ be a subsolution and a supersolution of problem (1.1), respectively, with $\underline{u} \leq \bar{u}$ a.e. in $\Omega$ and such that the Carathéodory function $f: \Omega \times \mathbb{R} \times \mathbb{R}^{n} \rightarrow \mathbb{R}$ fulfills

$$
|f(x, s, \xi)| \leq \sigma(x)+a \widetilde{E}^{-1}(A(|\xi|)) \text { a.e. } x \in \Omega \text {, all } s \in[\underline{u}(x), \bar{u}(x)], \text { all } \xi \in \mathbb{R}^{n},
$$

where $\sigma \in L^{\widetilde{A}_{n}}(\Omega), a>0$ and $E:\left[0,+\infty\left[\rightarrow\left[0,+\infty\left[\right.\right.\right.\right.$ is a Young function, $E \ll A_{n}$ near infinity. Assume also that $\underline{u}, \bar{u} \in L^{A_{n}}(\Omega)$.

Then problem $(P)$ possesses a solution $u \in W_{0}^{1, A}(\Omega)$ such that $\underline{u} \leq u \leq \bar{u}$ a.e. in $\Omega$.

In the proof of Theorems 3.2 (and 3.3), under one hypothesis, we construct a subsolution (a supersolution) via variational methods, a constant supersolution (a constant subsolution) and then we apply Theorem 3.6 to guarantee the existence of a solution.

Usually (see for instance [FMMT, MT]), the problem of finding a subsolution and a supersolution to (1.1) requires some additional assumptions on $f$ and sometimes on $\Omega$ too. A simple situation, different from that of Theorems 3.2 and 3.3, is one in which the structure of $f$ leads to constant sub and super solutions. In the examples below we specialize Theorem 3.6 (and consequently Theorems 3.2 and 3.3) to some classes of functions $A$, which govern the differential operator in the equation (1.1). We also consider functions $f$ for which the existence of a sub and of a super solution can easily be established. Even if such sub and supersolutions are bounded, the general assumptions on $\Omega$ prevent us to obtain the regularity of the solution too.

Obviously, all the growth conditions with respect to the gradient, considered in the examples below, are admissible also for functions $f$ satisfying (3.1) or (3.2), for which the existence of solution is guaranteed by Theorems 3.2 or 3.3 .

In all the examples $\Omega$ is an open subset in $\mathbb{R}^{n}$ with finite measure.

\subsection{The (p, q) - laplacian}

Consider the case when

$$
A(t)=\frac{t^{p}}{p}+\mu \frac{t^{q}}{q} \quad \text { for } t \geq 0
$$

where $1<q<p<\infty$ and $\mu>0$. Problem (1.1) then reads

$$
\begin{cases}-\operatorname{div}\left(\left(|\nabla u|^{p-2}+\mu|\nabla u|^{q-2}\right) \nabla u\right)=f(x, u, \nabla u) & \text { in } \Omega \\ u=0 & \text { on } \partial \Omega .\end{cases}
$$


We have

$$
A_{n}(t) \approx\left\{\begin{array}{ccc}
t^{p *} & \text { near infinity } & \text { if } p<n \\
e^{t^{n^{\prime}}} & \text { near infinity } & \text { if } p=n \\
+\infty & \text { near infinity } & \text { if } p>n
\end{array}\right.
$$

Also, if $p \leq n$ then (2.23) and (2.27) hold, while if $p>n$ then (2.28) holds.

Let $g: \mathbb{R} \rightarrow \mathbb{R}$ be a continuous function, such that $g\left(s_{1}\right)=g\left(s_{2}\right)=0$ for some $s_{1}<0<s_{2}$ and $g(s) \neq 0$ for all $s \in] s_{1}, s_{2}$ [. Take also a measurable sign changing function, $h: \Omega \rightarrow \mathbb{R}$.

Assume $p<n$ and $h \in L^{\left(p^{*}\right)^{\prime}}(\Omega)$. Let also $h_{1}:[0,1] \rightarrow \mathbb{R}$ be a continuous function such that $h(1)=\lg ^{-\frac{1}{p^{*}}}(2)$. Set

$$
f(x, s, \xi)=\left\{\begin{array}{cl}
g(s) h(x)+h_{1}(|\xi|) & \text { for }(x, s, \xi) \in \Omega \times \mathbb{R} \times \mathbb{R}^{n},|\xi| \leq 1 \\
g(s) h(x)+\frac{|\xi|}{\lg ^{\frac{1}{p^{*}}}\left(1+|\xi|^{*}\right)} & \text { for }(x, s, \xi) \in \Omega \times \mathbb{R} \times \mathbb{R}^{n},|\xi|>1 .
\end{array}\right.
$$

Then $u_{1}=s_{1}$ and $u_{2}=s_{2}$ are a subsolution and a supersolution to (3.7) and $u \equiv 0$ is not a solution nor a sub or a supersolution. Let $E(t)=\frac{t^{p *}}{\lg (1+t)}$. Then $\widetilde{E}^{-1}(t) \approx \frac{t^{\frac{1}{\left(p^{*}\right)^{\prime}}}}{\lg \frac{1}{p^{*}}(1+t)}$ near infinity, so we can find $a, b, c>0$ such that

$$
|f(x, s, \xi)| \leq b|h(x)|+c+a \widetilde{E}^{-1}(A(|\xi|)) \text { for } x \in \Omega, s \in\left[u_{1}, u_{2}\right], \xi \in \mathbb{R}^{n}
$$

and $f$ satisfies (3.5) with $\sigma(x)=b|h(x)|+c$. By Theorem 3.6, problem (3.7) has a nontrivial solution $u \in\left[u_{1}, u_{2}\right]$.

Let now $p \geq n$ and the functions $g$ and $h$ as above. Assume now $h \in L^{1}(\Omega)$ if $p>n, h \in L^{q}(\Omega)$, for some $q>1$, if $p=n$. Set

$$
f(x, s, \xi)=g(s) h(x)+\frac{|\xi|^{p}}{\lg \left(1+|\xi|^{p}\right)} \text { for }(x, s, \xi) \in \Omega \times \mathbb{R} \times \mathbb{R}^{n} .
$$

Then $u_{1}=s_{1}$ and $u_{2}=s_{2}$ are a subsolution and a supersolution to (3.7) and $u \equiv 0$ is not a solution nor a sub or supersolution. For $E(t)=e^{t}-1$ one has $\widetilde{E}^{-1}(t) \approx \frac{t}{\lg (1+t)}$ near infinity, so we can find $a, b, c>0$ such that

$$
|f(x, s, \xi)| \leq b|h(x)|+c+a(\widetilde{E})^{-1}(A(|\xi|)) \text { for } x \in \Omega, s \in\left[u_{1}, u_{2}\right], \xi \in \mathbb{R}^{n}
$$

and $f$ satisfies $(H)$ with $\sigma(x)=b|h(x)|+c$. By Theorem 3.6, problem (3.7) has a nontrivial solution $u \in\left[u_{1}, u_{2}\right]$.

Remark 3.7 The growth conditions on $f$ with respect to $\xi$ are weaker than those allowed in the classical Lebesgue spaces. Usually, when $p<n$ one has $|\xi|^{q}$, for some $q<\frac{p}{\left(p^{*}\right)^{\prime}}$, rather than $\frac{|\xi|^{\frac{p}{\left(p^{*}\right)^{\prime}}}}{\lg \frac{1}{p^{*}}\left(1+|\xi|^{p}\right)}$. Also, when $p \geq n$ the growth condition with respect to $\xi$ involves a power $|\xi|^{q}$, for some $q<p$ but this does not include $\frac{|\xi|^{p}}{\lg \left(1+|\xi|^{p}\right)}$. 


\subsection{The $\mathrm{A}(\mathbf{p}, \mathbf{q})$ - Laplacian}

Let us further consider the problem

$$
\begin{cases}-\operatorname{div}\left(\lg \left(1+|\nabla u|^{q}\right)|\nabla u|^{p-2} \nabla u\right)=f(x, u, \nabla u) & \text { in } \Omega \\ u=0 & \text { on } \partial \Omega,\end{cases}
$$

where $p>1, q>0$. Then (according to the convention on modify $A$ near 0 when (2.23) does not hold)

$$
A_{n}(t) \approx\left\{\begin{array}{cl}
t^{p *} \lg ^{\frac{n}{n-p}}(1+t) \text { near infinity } & \text { if } p<n \\
e^{\frac{n}{n-2}} \text { near infinity } & \text { if } p=n \\
+\infty \text { near infinity } & \text { if } p>n
\end{array}\right.
$$

Let $g: \mathbb{R} \rightarrow \mathbb{R}$ be a continuous function such that $g(0)<0$ and $g\left(s_{1}\right)=0$ for some $s_{1}<0$. Let $h: \Omega \rightarrow[0,+\infty[$ be a measurable function, $h(x)>0$ a.e. in $\Omega$.

Assume $p<n$ and let $h \in L^{\left(p^{*}\right)^{\prime}}(\Omega)$. Set

$$
f(x, s, \xi)=g(s) h(x)+|\xi|^{\frac{p}{\left(p^{*}\right)^{\prime}}} \lg \left(1+|\xi|^{p}\right) \text { for }(x, s, \xi) \in \Omega \times \mathbb{R} \times \mathbb{R}^{n} .
$$

Then $\underline{u}=s_{1}$ and $\bar{u} \equiv 0$ are a subsolution and a supersolution to (3.9), respectively and $u \equiv 0$ is not a solution. Let $E(t)=t^{p^{*}} \lg (1+t)$. Then $\widetilde{E}^{-1}(t) \approx t^{\frac{1}{\left(p^{*}\right)^{\prime}}} \lg ^{\frac{1}{p^{*}}}(1+t)$ near infinity, so we can find $a, b, c>0$ such that

$$
|f(x, s, \xi)| \leq b h(x)+c+a \widetilde{E}^{-1}(A(|\xi|)) \text { for } x \in \Omega, s \in\left[s_{1}, 0\right], \xi \in \mathbb{R}^{n},
$$

and $f$ satisfies $(H)$ with $\sigma(x)=b h(x)+c$. By Theorem 3.6, problem (3.9) has a nontrivial solution $u \in\left[s_{1}, 0\right]$.

Let now $p \geq n$ and $g$ and $h$ as above. Further, $h \in L^{1}(\Omega)$ if $p>n, h \in L^{q}(\Omega)$, for some $q>1$, if $p=n$. Set

$$
f(x, s, \xi)=g(s) h(x)+|\xi|^{p} \text { for }(x, s, \xi) \in \Omega \times \mathbb{R} \times \mathbb{R}^{n} .
$$

Then $\underline{u}=s_{1}$ and $\bar{u} \equiv 0$ are a subsolution and a supersolution to (3.7) respectively, and $u \equiv 0$ is not a solution. Let $E(t)=e^{t}-1$. Then $\widetilde{E}^{-1}(t) \approx \frac{t}{\lg (1+t)}$ near infinity, so we can find $a, b, c>0$ such that

$$
|f(x, s, \xi)| \leq b h(x)+c+a \widetilde{E}^{-1}(A(|\xi|)) \text { for } x \in \Omega, s \in\left[s_{1}, 0\right], \xi \in \mathbb{R}^{n},
$$

and $f$ satisfies $(H)$ with $\sigma(x)=b h(x)+c$. By Theorem 3.6, problem (3.6) has a nontrivial solution $u \in\left[s_{1}, 0\right]$.

\subsection{A general convex function $A$}

We examine now the problem

$$
\begin{cases}-\operatorname{div}\left(\frac{|\nabla u|^{p-2} \nabla u}{\lg ^{q}(1+|\nabla u|)}\right)=f(x, u, \nabla u) & \text { in } \Omega \\ u=0 & \text { on } \partial \Omega\end{cases}
$$

where $p>q+1 \geq 1$. Then (according to the convention on modify $A$ near 0 when $(2.23)$ does not hold)

$$
A_{n}(t) \approx\left\{\begin{array}{cc}
\frac{t^{p *}}{\lg \frac{n^{n}}{n-p}(1+t)} \text { near infinity } & \text { if } p<n \\
e^{t \frac{n}{q+n-1}} \text { near infinity } & \text { if } p=n \\
+\infty \text { near infinity } & \text { if } p>n
\end{array}\right.
$$


Let $g_{1}, g_{2}:\left[0,+\infty\left[\rightarrow\left[0,+\infty\left[\right.\right.\right.\right.$ be two unbounded, nondecreasing functions, such that $g_{1}(0)=g_{2}(0)=$ 0 . Let $a_{1}, a_{2}, c_{1}, c_{2}>0$.

Assume $p<n$. Let $\rho \in L^{\left(p^{*}\right)^{\prime}}(\Omega)$ and $f: \Omega \times \mathbb{R} \times \mathbb{R}^{n} \rightarrow \mathbb{R}$ be a Carathéodory function such that

$$
\begin{array}{r}
-c_{1}+g_{1}(|s|)-a_{1} \frac{|\xi|^{\left(p^{*}\right)^{\prime}}}{\lg ^{r}(1+|\xi|)} \leq f(x, s, \xi) \leq-c_{2}+g_{2}(|s|) \rho(x)+a_{2} \frac{|\xi|^{\left(p^{*}\right)^{\prime}}}{\lg ^{r}(1+|\xi|)} \\
\left.\qquad \text { for }(x, s, \xi) \in \Omega \times \mathbb{R} \times \mathbb{R}^{n} \text { and } r \in\right] q \frac{n+1}{n}, \frac{q}{\left(p^{*}\right)^{\prime}}+1[.
\end{array}
$$

If $k:=\inf \left\{s>0: g_{1}(s) \geq c_{1}\right\}$, then $\underline{u} \equiv-k$ is a subsolution to (3.10), and $\bar{u} \equiv 0$ is a supersolution but not a solution to (3.10). For the Young function $E(t)=\frac{t^{p^{*}}}{\lg ^{\lambda p^{*}}(1+t)}, \lambda=r-\frac{q}{\left(p^{*}\right)^{\prime}}$, one has $\widetilde{E}^{-1}(t) \approx \frac{t^{\frac{1}{\left(p^{*}\right)^{\prime}}}}{\lg ^{\lambda}(1+t)}$ near infinity, so we can find $a, b, c>0$ such that

$$
|f(x, s, \xi)| \leq b|\rho(x)|+c+a \widetilde{E}^{-1}(A(|\xi|)) \text { for } x \in \Omega, s \in[-k, 0], \xi \in \mathbb{R}^{n},
$$

and $f$ satisfies $(H)$ with $\sigma(x)=b|\rho(x)|+c$. By Theorem 3.6, problem (3.10) has a nontrivial solution $u \in[-k, 0]$.

Let now $p=n, \rho \in L^{t}(\Omega)$ for some $t>1$, and $f: \Omega \times \mathbb{R} \times \mathbb{R}^{n} \rightarrow \mathbb{R}$ be a Carathéodory function such that

$$
\begin{array}{r}
-c_{1}+g_{1}(|s|)-a_{1} \frac{|\xi|^{p}}{\lg ^{q+1}(1+|\xi|)} \leq f(x, s, \xi) \leq-c_{2}+g_{2}(|s|) \rho(x)+a_{2} \frac{|\xi|^{p}}{\lg ^{q+1}(1+|\xi|)} \\
\text { for }(x, s, \xi) \in \Omega \times \mathbb{R} \times \mathbb{R}^{n} .
\end{array}
$$

Then $\underline{u} \equiv-k, \bar{u} \equiv 0$ are a subsolution and a supersolution to (3.10) and $u \equiv 0$ is not a solution to (3.10). Taking $E(t)=e^{t}-1$ and arguing as in Example 3.2, we can prove that $f$ satisfies $(H)$. By Theorem 3.6, problem (3.10) has a nontrivial solution $u \in[-k, 0]$.

Under the same assumptions on the functions $g_{1}, g_{2}$ and on the constants $a_{1}, a_{2}, c_{1}, c_{2}$ listed above, the same arguments can be used to prove that problem (3.10) has a nontrivial solution $u \in[0, k]$, where $k:=\inf \left\{s>0: g_{2}(s) \geq c_{2}\right\}$, provided that $f: \Omega \times \mathbb{R} \times \mathbb{R}^{n} \rightarrow \mathbb{R}$ is a Carathéodory function such that

$$
\begin{array}{r}
c_{1}-g_{1}(|s|) \rho(x)-a_{1} \frac{|\xi|^{\left(p^{*}\right)^{\prime}}}{\lg ^{r}(1+|\xi|)} \leq f(x, s, \xi) \leq c_{2}-g_{2}(|s|)+a_{2} \frac{|\xi|^{\left(p^{*}\right)^{\prime}}}{\lg ^{r}(1+|\xi|)} \\
\left.\quad \text { for }(x, s, \xi) \in \Omega \times \mathbb{R} \times \mathbb{R}^{n}, \text { and } r \in\right] q \frac{n+1}{n}, \frac{q}{\left(p^{*}\right)^{\prime}}+1[\text { if } p<n
\end{array}
$$

or

$$
\begin{array}{r}
c_{1}-g_{1}(|s|) \rho(x)-a_{1} \frac{|\xi|^{p^{*}}}{\lg ^{q+1}(1+|\xi|)} \leq f(x, s, \xi) \leq c_{2}-g_{2}(|s|)+a_{2} \frac{|\xi|^{p^{*}}}{\lg ^{q+1}(1+|\xi|)} \\
\text { for }(x, s, \xi) \in \Omega \times \mathbb{R} \times \mathbb{R}^{n}, \text { if } p=n .
\end{array}
$$

\section{Auxiliary results}

This section is devoted to the statement of the technical results that we use in Section 5. Let's start by introducing the truncation operator that we need. For every $r \in \mathbb{R}$, we set $r^{+}=\max \{r, 0\}$, $r^{-}=\max \{-r, 0\}$. 
Let $\underline{u}, \bar{u} \in W^{1, A}(\Omega)$ be such that $(\bar{u})^{-}, \underline{u}^{+} \in W_{0}^{1, A}(\Omega)$, and $\underline{u} \leq \bar{u}$ a.e. in $\Omega$. The truncation operator $T: W_{0}^{1, A}(\Omega) \rightarrow W_{0}^{1, A}(\Omega)$ is defined by

$$
T(u)=\left\{\begin{array}{cc}
\bar{u} & \text { if } u>\bar{u} \\
u & \text { if } \underline{u} \leq u \leq \bar{u} \\
\underline{u} & \text { if } u<\underline{u}
\end{array}\right.
$$

The only hypothesis $\underline{u}, \bar{u} \in W^{1, A}(\Omega)$ does not guarantee that $T(u) \in W_{0}^{1, A}(\Omega)$. The properties of the truncating functions ensure that $T(u),(u-\bar{u})^{+},(u-\underline{u})^{-} \in W_{0}^{1, A}(\Omega)$. In particular $T$ is well defined. A different choice of $\underline{u}, \bar{u}$ (for instance $\underline{u}, \bar{u} \in L^{E}(\Omega)$ ) leads to $T: L^{E}(\Omega) \rightarrow L^{E}(\Omega)$. It is known (see $[\mathrm{H}]$, p.20) that

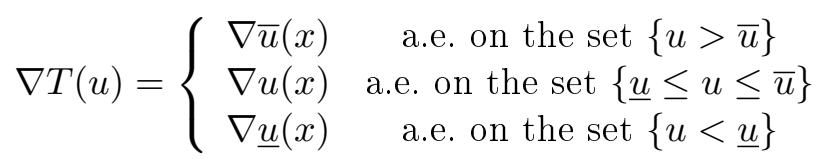

The following Lemma extends the continuity result on $T$ to the Orlicz-Sobolev spaces setting.

Lemma 4.1 If $A$ is a Young function, then the operator $T: W_{0}^{1, A}(\Omega) \rightarrow W_{0}^{1, A}(\Omega)$ defined by (4.1) is bounded (in the sense that it maps bounded sets into bounded sets). If $A \in \Delta_{2}$ near infinity, then it is continuous.

If $E$ is a Young function and $\bar{u}, \underline{u} \in L^{E}(\Omega)$, then $T: L^{E}(\Omega) \rightarrow L^{E}(\Omega)$ is bounded and continuous.

Proof. Let us prove that $T$ is bounded. Let $\mathcal{C} \subseteq W_{0}^{1, A}(\Omega)$ be a bounded set, and let $M_{0}$ be such that $\|u\|_{W_{0}^{1, A}(\Omega)} \leq M_{0}$ for all $u \in \mathcal{C}$. Take $\lambda=3 \max \left\{M_{0},\|\bar{u}\|_{W^{1, A}(\Omega)},\|\underline{u}\|_{W^{1, A}(\Omega)}\right\}$. Then from (4.2)

$$
\int_{\Omega} A\left(\frac{|\nabla T(u)|}{\lambda}\right) d x \leq \frac{1}{3} \int_{\Omega}\left[A\left(\frac{|\nabla u|}{M_{0}}\right)+A\left(\frac{|\nabla \bar{u}|}{\|\bar{u}\|_{W^{1, A}(\Omega)}}\right)+A\left(\frac{|\nabla \underline{u}|}{\|\underline{u}\|_{W^{1, A}(\Omega)}}\right)\right] d x \leq 1
$$

namely $\|T(u)\|_{W_{0}^{1, A}(\Omega)} \leq \lambda$ for all $u \in \mathcal{C}$.

Let $\left\{u_{k}\right\} \subset W_{0}^{1, A}(\Omega)$ be a sequence such that $u_{k} \rightarrow u$ in $W_{0}^{1, A}(\Omega)$. Then, up a subsequence, we can find $h \in L^{A}(\Omega)$ such that $\max \left\{\left|\nabla u_{k}(x)-\nabla u(x)\right|,\left|\nabla u_{k}(x)-\nabla \underline{u}(x)\right|,\left|\nabla u_{k}(x)-\nabla \bar{u}(x)\right|\right\} \leq h(x)$ for a.e. $x \in \Omega$, for all $k \in \mathbb{N}$. From (4.2)

$$
\left|\nabla T u_{k}(x)-\nabla T u(x)\right| \leq \max \{|\nabla \underline{u}(x)-\nabla u(x)|,|\nabla \bar{u}(x)-\nabla \underline{u}(x)|,|\nabla \bar{u}(x)-\nabla u(x)|, h(x)\}
$$
for a.e. $x \in \Omega$, all $k \in \mathbb{N}$.

Call $g(x)$ the right hand side of (4.4). Then $g \in L^{A}(\Omega)$ and since $A \in \Delta_{2}$ near infinity, from (2.11), $A\left(\frac{g(x)}{\lambda}\right) \in L^{1}(\Omega)$ for every $\lambda>0$. From $(2.21) \lim _{k \rightarrow+\infty}\left\|u_{k}-u\right\|_{W_{0}^{1,1}(\Omega)}=0$ and from Lemma 1.22 of $[\mathrm{H}] \lim _{k \rightarrow+\infty}\left\|T u_{k}-T u\right\|_{W^{1,1}(\Omega)}=0$. Thus $\lim _{k \rightarrow+\infty} \nabla T u_{k}=\nabla T u$ a.e. in $\Omega$. Moreover

$$
A\left(\frac{\left|\nabla T u_{k}(x)-\nabla T u(x)\right|}{\lambda}\right) \leq A\left(\frac{g(x)}{\lambda}\right) \text { for all } k \in \mathbb{N}
$$

so the conclusion follows via the Lebesgue theorem and (2.10).

Let now $E$ be as in the statement. Define $T$ is as in (4.1), but on the space $L^{E}(\Omega)$ and with the only assumption $\bar{u}, \underline{u} \in L^{E}(\Omega)$. Then $T$ is well defined, and its boundedness can be proved arguing as in (4.3). Let $\left\{u_{k}\right\} \subset L^{E}(\Omega)$ and $u \in L^{E}(\Omega)$, such that $\lim _{k \rightarrow+\infty}\left\|u_{k}-u\right\|_{L^{E}(\Omega)}=0$. Then

$$
\lim _{k \rightarrow+\infty} \int_{\Omega} E\left(\frac{\left|u_{k}(x)-u(x)\right|}{\lambda}\right) d x=0 \forall \lambda>0,
$$


and

$$
\left|T\left(u_{k}(x)\right)-T(u(x))\right| \leq\left|u_{k}(x)-u(x)\right| \text { a.e } x \in \Omega, \forall k \in \mathbb{N},
$$

so

$$
\lim _{k \rightarrow+\infty} \int_{\Omega} E\left(\frac{\left|T\left(u_{k}(x)\right)-T(u(x))\right|}{\lambda}\right) d x=0 \forall \lambda>0 .
$$

The continuity is thus a consequence of $(2.9)$ and (2.10).

In the sequel, unlike the usual notation, we denote with $[\underline{u}, \bar{u}]$ the following subset of $W_{0}^{1, A}(\Omega)$ associated to an ordered pair $\underline{u} \leq \bar{u}$ of functions in $W^{1, A}(\Omega)$ :

$$
[\underline{u}, \bar{u}]:=\left\{u \in W_{0}^{1, A}(\Omega): \underline{u}(x) \leq u(x) \leq \bar{u}(x) \text { a.e. } x \in \Omega\right\} .
$$

We introduce now the hypotheses on $f$. They involve two functions like those in (4.1). This is essential in order to then define the Nemitskyl operator associated to $f$ in the interval $[\underline{u}, \bar{u}] \subset$ $W_{0}^{1, A}(\Omega)$. They are the same as those already seen in Theorem 3.6, except that here the functions $\underline{u}, \bar{u}$ do not need to be a sub and a supersolution. This is due to the fact that all the technical results of this section hold true without that hypotheses.

We assume that $f: \Omega \times \mathbb{R} \times \mathbb{R}^{n} \rightarrow \mathbb{R}$ is a Carathéodory function and satisfies the following growth condition:

$(\mathrm{H})$ there exists a Young function $E, E \ll A_{n}$ near infinity, a function $\sigma \in L^{\tilde{A}_{n}}(\Omega)$, a constant $a>0$ and two functions $\underline{u}, \bar{u} \in W^{1, A}(\Omega)$, with the properties $(\bar{u})^{-}, \underline{u}^{+} \in W_{0}^{1, A}(\Omega)$, and $\underline{u} \leq \bar{u}$ a.e. in $\Omega$, such that

$$
|f(x, s, \xi)| \leq \sigma(x)+a \widetilde{E}^{-1}(A(|\xi|)) \text { a.e. } x \in \Omega \text {, all } s \in[\underline{u}(x), \bar{u}(x)], \text { all } \xi \in \mathbb{R}^{n} .
$$

Lemma 4.2 Let $A$ be a Young function, $A \in \Delta_{2}$ near infinity, for which (2.23) and (2.27), or (2.28) hold. Assume that $f: \Omega \times \mathbb{R} \times \mathbb{R}^{n} \rightarrow \mathbb{R}$ satisfies $(H)$.

(i) If $\left\{u_{k}\right\} \subset W_{0}^{1, A}(\Omega)$ is a sequence weakly converging to $u \in W_{0}^{1, A}(\Omega)$, then

$$
\lim _{k \rightarrow \infty} \int_{\Omega} f\left(x, T u_{k}, \nabla T u_{k}\right)\left(u_{k}-u\right) d x=0
$$

(ii) If $\left\{u_{k}\right\} \subset W_{0}^{1, A}(\Omega)$ is a sequence converging to $u \in W_{0}^{1, A}(\Omega)$, then

$$
\lim _{k \rightarrow \infty}\left\|f\left(x, T u_{k}, \nabla T u_{k}\right)-f(x, T u, \nabla T u)\right\|_{L^{\widetilde{A}_{n}(\Omega)}}=0 .
$$

Proof. $(i)$ Let $\left\{u_{k}\right\}$ be as in $(i)$. Then $\left\{u_{k}\right\}$ converges weakly to $u$ in $L^{B}(\Omega)$ and strongly in $L^{E}(\Omega)$ (because $E \ll A_{n}$ near infinity). The sequence $\left\{\nabla T u_{k}\right\}$ is bounded in $L^{A}(\Omega)$, so from (2.18) there exists $c>0$ such that

$$
\left\|\widetilde{E}^{-1}\left(A\left(\left|\nabla T u_{k}\right|\right)\right)\right\|_{L^{\widetilde{E}}(\Omega)} \leq \max \{1, c\} \text { for all } k \in \mathbb{N} .
$$

By definition of weak convergence

$$
\lim _{k \rightarrow \infty} \int_{\Omega} \sigma(x)\left(u_{k}-u\right) d x=0 .
$$

Using $(H)$ and $(2.8)$

$$
\begin{array}{r}
\left|\int_{\Omega} f\left(x, T u_{k}(x), \nabla T u_{k}(x)\right)\left(u_{k}-u\right) d x\right| \leq\left|\int_{\Omega} \sigma(x)\left(u_{k}-u\right) d x\right|+a \int_{\Omega} \widetilde{E}^{-1}\left(A\left(\left|\nabla T u_{k}(x)\right|\right)\left|u_{k}-u\right| d x\right. \\
\leq\left|\int_{\Omega} \sigma(x)\left(u_{k}-u\right) d x\right|+2 a\left\|\widetilde{E}^{-1}\left(A\left(\left|\nabla T u_{k}(x)\right|\right)\right)\right\|_{L^{\widetilde{E}}(\Omega)}\left\|u_{k}-u\right\|_{L^{E}(\Omega)} .
\end{array}
$$


Taking into account (4.7) and (4.8), equation (4.6) follows from the inequality above. (ii) Let $\left\{u_{k}\right\}$ as in $(i i)$. By the definition of Luxemburg norm it suffices to show that

$$
\lim _{k \rightarrow \infty} \int_{\Omega} \widetilde{A}_{n}\left(\frac{\left|f\left(x, T u_{k}, \nabla T u_{k}\right)-f(x, T u, \nabla T u)\right|}{\lambda}\right) d x=0 \quad \text { for every } \lambda>0 .
$$

By the convexity of $\widetilde{A}_{n}$ and $(H)$

$$
\begin{aligned}
\widetilde{A}_{n}\left(\frac{\left|f\left(x, T u_{k}, \nabla T u_{k}\right)-f(x, T u, \nabla T u)\right|}{\lambda}\right) & \leq \frac{1}{2} \widetilde{A}_{n}\left(\frac{4 \sigma(x)}{\lambda}\right) \\
+\frac{1}{4} \widetilde{A}_{n}\left(\frac{4 a \widetilde{E}^{-1}\left(A\left(\left|\nabla T\left(u_{k}(x)\right)\right|\right)\right)}{\lambda}\right) & +\frac{1}{4} \widetilde{A}_{n}\left(\frac{4 a \widetilde{E}^{-1}(A(|\nabla T(u(x))|))}{\lambda}\right)
\end{aligned}
$$

for a.e. $x \in \Omega$, all $k \in \mathbb{N}$. Since $E \ll A_{n}$, from (2.3), there exists $t_{0} \geq 0$ such that

$$
\widetilde{E}^{-1}(A(t)) \leq \frac{\lambda}{4 a} \widetilde{A}_{n}^{-1}(A(t)), \quad \text { if } t>t_{0}
$$

Proposition 2.30 guarantees that $\widetilde{A}_{n} \in \Delta_{2}$ at infinity. As $\sigma \in L^{\widetilde{A}_{n}}(\Omega)$

$$
\widetilde{A}_{n}\left(\frac{4 \sigma(x)}{\lambda}\right) \in L^{1}(\Omega) \text { for every } \lambda>0 .
$$

Choosing $t=\left|\nabla T\left(u_{k}(x)\right)\right|$ and $t=|\nabla T(u(x))|$ in (4.10)

$$
\begin{gathered}
\widetilde{A}_{n}\left(\frac{4 a}{\lambda} \widetilde{E}^{-1}\left(A\left(\left|\nabla T\left(u_{k}(x)\right)\right|\right)\right)\right) \leq \widetilde{A}_{n}\left(\frac{4 a}{\lambda} \widetilde{E}^{-1}\left(A\left(t_{0}\right)\right)\right)+A\left(\left|\nabla T\left(u_{k}(x)\right)\right|\right) \quad \text { for a.e. } x \in \Omega \\
\widetilde{A}_{n}\left(\frac{4 a}{\lambda} \widetilde{E}^{-1}(A(|\nabla T(u(x))|))\right) \leq \widetilde{A}_{n}\left(\frac{4 a}{\lambda} \widetilde{E}^{-1}\left(A\left(t_{0}\right)\right)\right)+A(|\nabla T((x))|) \quad \text { for a.e. } x \in \Omega
\end{gathered}
$$

From Lemma 4.1 , the sequence $\left\{T u_{k}\right\}$ converges to $T u$ in $W_{0}^{1, A}(\Omega)$. Then

$$
\lim _{k \rightarrow \infty} T u_{k}(x)=T u(x), \text { and } \lim _{k \rightarrow \infty} \nabla T u_{k}(x)=\nabla T u(x) \text { a.e. in } \Omega .
$$

Let $g \in L^{A}(\Omega)$ be such that $\left|T u_{k}(x)\right| \leq g(x)$ and $\left|\nabla T u_{k}(x)\right| \leq g(x)$ a.e. in $\Omega$ and for all $k \in \mathbb{N}$. Using (4.11), (4.12) and (4.13) in (4.9)

$$
\begin{gathered}
\widetilde{A}_{n}\left(\frac{\left|f\left(x, T u_{k}, \nabla T u_{k}\right)-f(x, T u, \nabla T u)\right|}{\lambda}\right) \leq \frac{1}{2}\left(\widetilde{A}_{n}\left(\frac{4 \sigma(x)}{\lambda}\right)+\widetilde{A}_{n}\left(\frac{4 a}{\lambda} \widetilde{E}^{-1}\left(A\left(t_{0}\right)\right)\right)\right) \\
\left.+\frac{1}{4} A(g(x))\right)+\frac{1}{4} A(|\nabla T(u(x))|) \quad \text { for a.e. } x \in \Omega, \text { for } k \in \mathbb{N} .
\end{gathered}
$$

Thanks to $|\Omega|<\infty,(4.11)$ and $A \in \Delta_{2}$ near infinity, the right-hand side of (4.15) is a function in $v_{\lambda} \in L^{1}(\Omega)$. We proved that, for every $\lambda>0$, there exists a function $v_{\lambda} \in L^{1}(\Omega)$ such that

$$
\widetilde{A}_{n}\left(\frac{\left|f\left(x, T u_{k}, \nabla T u_{k}\right)-f(x, T u, \nabla T u)\right|}{\lambda}\right) \leq v_{\lambda}(x) \text { for a.e. } x \in \Omega \text {, for } k \in \mathbb{N} \text {. }
$$

Taking into account (4.14) the conclusion follows via the classical dominated convergence theorem. 
sky

Hypothesis $(H)$ enables us to consider the composition of the Nemitskyi operator associated with $f$ with the operator $T$ as in (4.1).

We denote by $\left(W_{0}^{1, A}(\Omega)\right)^{*}$ the dual space of $W_{0}^{1, A}(\Omega)$ and by $\langle.,$.$\rangle the duality paring between them.$

Proposition 4.3 Let $A$ be a Young function, $A \in \Delta_{2}$ near infinity, satisfying (2.23) and (2.27), or (2.28). Assume that $f: \Omega \times \mathbb{R} \times \mathbb{R}^{n} \rightarrow \mathbb{R}$ satisfies $(H)$. Then the operator $\mathcal{N}_{f} \circ T: W_{0}^{1, A}(\Omega) \rightarrow$ $\left(W_{0}^{1, A}(\Omega)\right)^{*}$, given by

$$
\left\langle\mathcal{N}_{f} \circ T(u), v\right\rangle=\int_{\Omega} f(x, T u, \nabla T u) v(x) d x
$$

is well defined, bounded and continuous.

Proof. Arguing as for (4.7), we know that $\widetilde{E}^{-1}(A(|\nabla T u(x)|)) \in L^{\widetilde{E}}(\Omega)$ for all $u \in W_{0}^{1, A}(\Omega)$. Using $(H),(2.8),(2.12)$ and $(2.26)$

$$
\begin{aligned}
\mid \int_{\Omega} f(x, T u(x), \nabla T u(x)) & v(x) d x|\leq| \int_{\Omega} \sigma(x) v(x) d x \mid+a \int_{\Omega} \widetilde{E}^{-1}(A(|\nabla T u(x)|)|v(x)| d x \\
\leq & 2\|\sigma\|_{L^{\widetilde{B}}(\Omega)}\|v\|_{L^{B}(\Omega)}+2 a\left\|E^{-1}(A(|\nabla T u(x)|))\right\|_{L^{\widetilde{E}}(\Omega)}\|v\|_{L^{E}(\Omega)} \\
& \leq 2 C\left(\|\sigma\|_{L^{\widetilde{B}(\Omega)}}+a\left\|E^{-1}(A(|\nabla T u(x)|))\right\|_{L^{\widetilde{E}}(\Omega)}\right)\|v\|_{W_{0}^{1, A}(\Omega)} .
\end{aligned}
$$

for every $u, v \in W_{0}^{1, A}(\Omega)$, so $\mathcal{N}_{f} \circ T$ is well defined. The same arguments show that if $\mathcal{C}$ is any bounded subset of $W_{0}^{1, A}(\Omega)$ then

$$
\left\|\mathcal{N}_{f} \circ T(u)\right\|_{\left(W_{0}^{1, A}(\Omega)\right)^{*}} \leq 2 C\left(\|\sigma\|_{L^{\widetilde{B}}(\Omega)}+a c_{1}\right) \quad \text { for every } u \in \mathcal{C},
$$

with the constant $c_{1}=\max \{1, c\}$ given by (2.18), thus $\mathcal{N}_{f} \circ T$ is bounded.

The continuity of $\mathcal{N}_{f} \circ T$ is a consequence of Lemma 4.2, part (ii).

By means of the Young function $E \ll A_{n}$ and of the functions $\underline{u}, \bar{u}$ as in in hypothesis $(\mathrm{H})$, we introduce the cut-off function $\pi: \Omega \times \mathbb{R} \rightarrow \mathbb{R}$

$$
\pi(x, s)=\left\{\begin{array}{cc}
\widetilde{E}^{-1}(E(s-\bar{u}(x)) & \text { if } s>\bar{u}(x) \\
0 & \text { if } \underline{u}(x) \leq s \leq \bar{u}(x) \\
-\widetilde{E}^{-1}(E(\underline{u}(x)-s)) & \text { if } s<\underline{u}(x) .
\end{array}\right.
$$

After, consider

$$
\pi(\cdot, u(\cdot))=\widetilde{E}^{-1}(E(|u(\cdot)-T u(\cdot)|)) \operatorname{sign}(u(\cdot)-T u(\cdot)) \text { for } u \in W_{0}^{1, A}(\Omega) .
$$

We know that $u-T u \in W_{0}^{1, A}(\Omega)$ and thus in $L^{A_{n}}(\Omega)$, so (2.18) (with $C=A_{n}, D=E$ ) allows to state that $\pi(\cdot, u(\cdot)) \in L^{\widetilde{E}}(\Omega)$ for all $u \in W_{0}^{1, A}(\Omega)$. Other properties of $\pi$ are collected in the Lemma below.

Lemma 4.4 Let $\pi$ be defined by (4.16).

(i) Let $\left\{u_{k}\right\} \subset W_{0}^{1, A}(\Omega)$ be a sequence weakly converging to $u \in W_{0}^{1, A}(\Omega)$. Then

$$
\lim _{k \rightarrow \infty} \int_{\Omega} \pi\left(x, u_{k}(x)\right)\left(u_{k}-u\right) d x=0 .
$$

(ii) Assume that $A \in \Delta_{2}$ near infinity or $\underline{u}, \bar{u} \in L^{A_{n}}(\Omega)$. Let $\left\{u_{k}\right\} \subset W_{0}^{1, A}(\Omega)$ be a sequence converging to $u \in W_{0}^{1, A}(\Omega)$, then

$$
\lim _{k \rightarrow \infty}\left\|\pi\left(\cdot, u_{k}(\cdot)\right)-\pi(\cdot, u(\cdot))\right\|_{L^{\widetilde{A}_{n}(\Omega)}}=0 .
$$


Proof. (i) By (4.16) and (2.8)

$$
\begin{aligned}
\left|\int_{\Omega} \pi\left(x, u_{k}(x)\right)\left(u_{k}-u\right) d x\right| \leq \int_{\Omega} \widetilde{E}^{-1}\left(E\left(\left|u_{k}(x)-T u_{k}(x)\right|\right)\left|u_{k}-u\right| d x\right. \\
\leq 2 \| \widetilde{E}^{-1}\left(E\left(\left|u_{k}-T u_{k}\right|\right)\left\|_{L^{\widetilde{E}}(\Omega)}\right\| u_{k}-u \|_{L^{E}(\Omega)} .\right.
\end{aligned}
$$

Since the sequence $\left\{u_{k}-T u_{k}\right\}$ is bounded in $L^{A_{n}}(\Omega)$, and $E \ll A_{n}$, by $(2.18)$ there exists a constant $c>0$ such that

$$
\left\|\widetilde{E}^{-1}\left(E\left(\left|u_{k}-T u_{k}\right|\right)\right)\right\|_{L^{\widetilde{E}}(\Omega)} \leq \max \{1, c\}
$$

and (4.17) follows.

(ii) By the definition of Luxemburg norm it suffices to show that

$$
\lim _{k \rightarrow \infty} \int_{\Omega} \widetilde{A}_{n}\left(\frac{\left|\pi\left(x, u_{k}(x)\right)-\pi(x, u(x))\right|}{\lambda}\right) d x=0 \quad \text { for every } \lambda>0 .
$$

From Lemma 4.1, the sequence $\left\{u_{k}-T\left(u_{k}\right)\right\}$ converges to $u-T(u)$ in $W_{0}^{1, A}(\Omega)$ and thus in $L^{A_{n}}(\Omega)$. Arguing as in Lemma 4.2, there exists $v \in L^{A_{n}}(\Omega)$ and $t_{0}>0$ such that

$$
\begin{gathered}
\widetilde{A}_{n}\left(\frac{\left|\pi\left(x, u_{k}(x)\right)-\pi(x, u(x))\right|}{\lambda}\right) \leq \widetilde{A}_{n}\left(\frac{2}{\lambda} \widetilde{E}^{-1}\left(E\left(t_{0}\right)\right)\right) \\
+\frac{1}{2}[E(v(x))+E(|u(x)-T(u(x))|)] \quad \text { for a.e. } x \in \Omega \text {, for } k \in \mathbb{N} .
\end{gathered}
$$

Since $u, v \in L^{A_{n}}(\Omega)$, and $E \ll A_{n}$ the right-hand side of (4.18) is a function in $v_{\lambda} \in L^{1}(\Omega)$. We proved that, for every $\lambda>0$, there exists a function $v_{\lambda} \in L^{1}(\Omega)$ such that

$$
\widetilde{A}_{n}\left(\frac{\left|\pi\left(x, u_{k}(x)\right)-\pi(x, u(x))\right|}{\lambda}\right) \leq v_{\lambda}(x) \text { for a.e. } x \in \Omega, \text { for } k \in \mathbb{N} \text {. }
$$

Moreover,

$$
\lim _{k \rightarrow \infty} \pi\left(x, u_{k}(x)\right)=\pi(x, u(x)) \text { for a.e. } x \in \Omega,
$$

and the conclusion follow via the classical dominated convergence theorem.

We are now in position to analyze the Nemytskij operator associated to $\pi$.

Proposition 4.5 Let $\Omega$ be an open set in $\mathbb{R}^{n}$ such that $|\Omega|<\infty$, and let $A$ be a Young function, satisfying (2.23) and (2.27) or (2.28). Then $\Pi: W_{0}^{1, A}(\Omega) \rightarrow\left(W_{0}^{1, A}(\Omega)\right)^{*}$, given by

$$
\Pi(u)(v)=\int_{\Omega} \pi(x, u(x)) v(x) d x
$$

is well defined, bounded. It is also continuous, provided $\underline{u}, \bar{u} \in L^{A_{n}}(\Omega)$ or $A \in \Delta_{2}$ near infinity.

Proof. We have previously observed that $\pi(\cdot, u(\cdot)) \in L^{\widetilde{E}}(\Omega)$. Also

$$
\int_{\Omega}|\pi(x, u(x)) v(x)| d x \leq 2\|\pi(\cdot, u(\cdot))\|_{L^{\widetilde{E}}(\Omega)}\|v\|_{L^{E}(\Omega)} \leq 2 C\|\pi(\cdot, u(\cdot))\|_{L^{\widetilde{E}}(\Omega)}\|v\|_{W_{0}^{1, A}(\Omega)}
$$

for every $u \in W_{0}^{1, A}(\Omega)$. Thus $\Pi$ is well defined and bounded.

The continuity of $\Pi$ follows from Lemma 4.4 part (ii). 
Lemma 4.6 Let $\Pi$ be defined as in (4.19). If $\underline{u}, \bar{u} \in L^{A_{n}}(\Omega)$, there exists a positive constant $c_{2}=$ $c_{2}(\underline{u}, \bar{u}, E)$ such that

$$
\Pi(u)(u) \geq 2 \int_{\Omega} E\left(\frac{|u|}{2}\right)-c_{2} \quad \text { for all } u \in L^{E}(\Omega) .
$$

Proof. The proof is a simply calculation based on (2.5), on the monotonicity of $E$ and of $\widetilde{E}^{-1}$ and on this inequality that holds for a general convex function $C$ :

$$
C\left(\frac{|t|}{2}\right) \leq \frac{1}{2} C(|t|-|s|)+\frac{1}{2} C(|s|) \text { for all } t, s \in \mathbb{R}, \text { such that }|t|>|s| .
$$

We distinguish all the situations that may occur.

- If $u(x)<\underline{u}(x) \leq 0$ then

$$
\pi(u) u=\widetilde{E}^{-1}(E(\underline{u}-u))(-u) \geq \frac{E(\underline{u}-u)}{\underline{u}-u}(-u)>E(\underline{u}-u) \geq 2 E\left(\frac{|u|}{2}\right)-E(|\underline{u}|) .
$$

- If $u(x)<0<\underline{u}(x)$ then

$$
\pi(u) u=\widetilde{E}^{-1}(E(\underline{u}-u))(-u) \geq \widetilde{E}^{-1}(E(u))(-u) \geq E(-u)=E(|u|) .
$$

- If $0 \leq u(x)<\underline{u}(x)$ then $\widetilde{E}^{-1}(E(\underline{u}-u)) \leq \widetilde{E}^{-1}\left(E_{n}(v)\right) \leq 2 \frac{E(\underline{u})}{\underline{u}} \leq 2\left(\frac{E(\underline{u})}{u}\right)$, thus $\pi(u) u=\widetilde{E}^{-1}(E(\underline{u}-u))(-u)>-2 E(\underline{u})$ and from $E(u)-E(\underline{u})<0$

$$
\pi(u) u>-3 E(\underline{u})+E(u) .
$$

- If $\underline{u}(x) \leq u(x) \leq \bar{u}(x)$ then $\pi(u)=0$ and $E(|u|) \leq \max \{E(|\underline{u}|), E(|\bar{u}|)\} \leq E(|\underline{u}|)+E(|\bar{u}|)$, thus

$$
E(|u|)-E(|\underline{u}|)-E(|\bar{u}|)<0=\pi(u) u
$$

- If $0 \leq \bar{u}(x)<u(x)$ then

$$
\pi(u) u=\widetilde{E}^{-1}(E(u-\bar{u})) u \geq \frac{E(u-\bar{u})}{u-\bar{u}} u>E(u-\bar{u}) \geq 2 E\left(\frac{|u|}{2}\right)-E(|\bar{u}|) .
$$

- If $\bar{u}(x)<0<u(x)$ then

$$
\pi(u) u=\widetilde{E}^{-1}(E(u-\bar{u})) u \geq \widetilde{E}^{-1}(E(u)) u \geq E(u) .
$$

- If $\bar{u}(x)<u(x) \leq 0$ then $\widetilde{E}^{-1}(E(u-\bar{u})) \leq(\widetilde{E})^{-1}(E(|\bar{u}|)) \leq 2 \frac{E(|\bar{u}|)}{|\bar{u}|} \leq 2 \frac{E(|\bar{u}|)}{|u|}$, thus $\pi(u) u=$ $\widetilde{E}^{-1}(E(u-\bar{u})) u>-2 E(|\bar{u}|)$ and from $E(|u|)<E(|\bar{u}|)$

$$
\pi(u) u>-3 E(|\bar{u}|)+E(|u|) .
$$

Put $\Omega_{1}=\{u<\underline{u} \leq 0\} \bigcup\{0 \leq \bar{u}<u\}$, then

$$
\begin{gathered}
\Pi(u) u \geq \int_{\Omega_{1}} 2 E\left(\frac{|u|}{2}\right) d x+\int_{\Omega \backslash \Omega_{1}} E(|u|) d x-\left(\int_{\{u<\underline{u} \leq 0\}} E(|\underline{u}|) d x+\int_{\{0<u<\underline{u}\}} 3 E(|\underline{u}|) d x\right)+ \\
-\left(\int_{\{\underline{u} \leq u \leq \bar{u}\}}(E(|\underline{u}|)+E(|\bar{u}|)) d x+\int_{\{0 \leq \bar{u}<u\}} E(|\bar{u}|) d x+\int_{\{\bar{u}<u \leq 0\}} 3 E(|\bar{u}|) d x\right) \\
\geq 2 \int_{\Omega} E\left(\frac{|u|}{2}\right) d x-3 \int_{\Omega}(E(|\underline{u}|)+E(|\bar{u}|)) d x
\end{gathered}
$$

The assumptions $\underline{u}, \bar{u} \in L^{A_{n}}(\Omega)$ and $E \ll A_{n}$ guarantee that $3 \int_{\Omega}(E(|\underline{u}|)+E(|\bar{u}|)) d x<c_{2}$, for some $c_{2}>0$ so

$$
\Pi(u) u \geq 2 \int_{\Omega} E\left(\frac{|u|}{2}\right) d x-c_{2} .
$$


Let us now consider the operator $S: W_{0}^{1, A}(\Omega) \rightarrow\left(W_{0}^{1, A}(\Omega)\right)^{*}$, defined as

$$
\langle S u, v\rangle=\int_{\Omega} A^{\prime}(|\nabla u|) \frac{\nabla u}{|\nabla u|} \cdot \nabla v d x
$$

for $u, v \in W_{0}^{1, A}(\Omega)$. Important properties of the operator $S$ are listed in the next proposition.

Proposition 4.7 Assume that $A \in C^{1}([0,+\infty[)$ is a strictly convex, Young function, satisfying (2.14) and $A^{\prime}(0)=0$. Then the operator $S: W_{0}^{1, A}(\Omega) \rightarrow\left(W_{0}^{1, A}(\Omega)\right)^{*}$, introduced in $(4.21)$ is well defined, bounded and continuous.

Proof. The condition $A^{\prime}(0)=0$ guarantees that $\lim _{\xi \rightarrow 0} A^{\prime}(|\xi|) \frac{\xi}{|\xi|}=0$ so the integral is defined also when $|\nabla u|=0$. Also $i_{A}>1$ guarantees that $\lim _{t \rightarrow+\infty} \frac{A(t)}{t}=+\infty$ so (A23) of [BC] leads to $A^{\prime}(|\nabla u|) \frac{\nabla u}{|\nabla u|} \in L^{\widetilde{A}}(\Omega)$ and from $(2.8)$

$$
|\langle S u, v\rangle| \leq 2\left\|A^{\prime}(|\nabla u|) \frac{\nabla u}{|\nabla u|}\right\|_{L^{\widetilde{A}}(\Omega)}\|\nabla v\|_{L^{A}(\Omega)} .
$$

Similarly, if $\mathcal{C} \subseteq W_{0}^{1, A}(\Omega)$ is such that $\|u\|_{W_{0}^{1, A}(\Omega)} \leq M_{0}$ for some $M_{0} \in \mathbb{R}$ then

$$
\int_{\Omega} \widetilde{A}\left(A^{\prime}\left(\frac{|\nabla u|}{2 M_{0}}\right) \frac{\nabla u}{|\nabla u|}\right) d x \leq \int_{\Omega} A\left(\frac{|\nabla u|}{M_{0}}\right) d x \leq 1
$$

So

$$
\left\|A^{\prime}(|\nabla u|) \frac{\nabla u}{|\nabla u|}\right\|_{L^{\widetilde{A}}(\Omega)} \leq 2 M_{0}
$$

The continuity is proved in [BC] Proposition $4.1($ take $\Phi(\xi)=A(|\xi|))$.

We conclude the Section recalling the main theorem on peudomonotone operators.

Definition 4.8 A mapping $A: X \rightarrow X^{*}$ is called

(i) coercive if $\lim _{\|u\| \rightarrow \infty} \frac{\langle A u, u\rangle}{\|u\|}=+\infty$;

(ii) pseudomonotone if $u_{n} \rightarrow u$ and $\limsup _{n \rightarrow+\infty}\left\langle A u_{n}, u_{n}-u\right\rangle \leq 0$ imply that $A u_{n} \rightarrow A u$ and $\left\langle A u_{n}, u_{n}\right\rangle \rightarrow\langle A u, u\rangle$.

Theorem 4.9 (see [CLM, Theorem 2.99]) Let $X$ be a real reflexive Banach space and let $A: X \rightarrow$ $X^{*}$ be a bounded, coercive and pseudomonotone operator. Then, for every $b \in X^{*}$ the equation $A x=b$ has at least solution $x \in X$.

\section{Proof of the Main results}

This Section is devoted to the proof of the main results. We perturb problem (1.1) with the Nemytskij operator $\Pi$ defined in (4.19), times a parameter $\lambda>0$, truncate $f$ too and formulate an auxiliary problem associated to (1.1).

$$
\begin{cases}-\operatorname{div}\left(A^{\prime}(|\nabla u|) \frac{\nabla u}{|\nabla u|}\right)+\lambda \Pi(u)=N_{f}(T u) & \text { in } \Omega, \\ u=0 & \text { on } \partial \Omega .\end{cases}
$$

The solvability of problem (5.1) can be guaranteed provided the parameter $\lambda>0$ is sufficiently large as shown in the following result. 
Theorem 5.1 Let $\Omega$ be an open set in $\mathbb{R}^{n}$, with $n \geq 2$, such that $|\Omega|<\infty$. Let $A \in C^{1}([0,+\infty))$ be a Young function for which (2.16) holds. Assume that $A$ satisfies (2.23) and (2.27), or (2.28), and that the Carathéodory function $f: \Omega \times \mathbb{R} \times \mathbb{R}^{n} \rightarrow \mathbb{R}$ fulfills hypothesis $(H)$. Assume also that $\underline{u}, \bar{u} \in L^{A_{n}}(\Omega)$.

Then there exists $\lambda_{0}>0$ such that (5.1) admits a solution whenever $\lambda \geq \lambda_{0}$.

Proof. For all $\lambda>0$ consider the operator $\mathcal{A}_{\lambda}: W_{0}^{1, A}(\Omega) \rightarrow\left(W_{0}^{1, A}(\Omega)\right)^{*}$, defined by

$$
\begin{array}{r}
\left\langle\mathcal{A}_{\lambda}(u), v\right\rangle=\int_{\Omega} A^{\prime}(|\nabla u|) \frac{\nabla u}{|\nabla u|} \cdot \nabla v d x+\lambda \int_{\Omega} \pi(x, u) v d x-\int_{\Omega} f(x, T u, \nabla T u) v d x= \\
\left\langle S u+\lambda \Pi u-\mathcal{N}_{f} \circ T(u), v\right\rangle \quad \text { for } u, v \in W_{0}^{1, A}(\Omega) .
\end{array}
$$

We prove that $\mathcal{A}_{\lambda}$ is well defined, bounded, pseudomonotone and there is $\lambda_{0}>0$ such that $\mathcal{A}_{\lambda}$ is coercive for all $\lambda>\lambda_{0}$.

Due to Propositions 4.3, 4.5 and $4.7, \mathcal{A}_{\lambda}$ is well defined, bounded and continuous. To prove that it is pseudomonotone, we take $u \in W_{0}^{1, A}(\Omega)$, and a sequence $\left\{u_{k}\right\} \subset W_{0}^{1, A}(\Omega)$ such that

$$
u_{k} \rightarrow u \quad \text { in } W_{0}^{1, A}(\Omega), \text { and } \limsup _{k \rightarrow \infty}\left\langle\mathcal{A}_{\lambda}\left(u_{k}\right), u_{k}-u\right\rangle \leq 0 .
$$

Then $u_{k} \rightarrow u$ in $L^{E}(\Omega)$ and it is bounded in $L^{A_{n}}(\Omega)$, so (4.6) and (4.17) allow to write

$$
\limsup _{k \rightarrow \infty}\left\langle S\left(u_{k}\right), u_{k}-u\right\rangle \leq 0
$$

Thus (see [BC], Lemma 4.6) $u_{k} \rightarrow u$ in $W_{0}^{1, A}(\Omega)$ and consequently

$$
\lim _{k \rightarrow \infty}\left\|\mathcal{A}_{\lambda}\left(u_{k}\right)-\mathcal{A}_{\lambda}(u)\right\|_{\left(W_{0}^{1, A}(\Omega)\right)^{*}}=0
$$

so $\left\langle\mathcal{A}_{\lambda}\left(u_{k}\right), u_{k}\right\rangle \rightarrow\left\langle\mathcal{A}_{\lambda}(u), u\right\rangle,\left\langle\mathcal{A}_{\lambda}\left(u_{k}\right), v\right\rangle \rightarrow\left\langle\mathcal{A}_{\lambda}(u), v\right\rangle$ for all $v \in W_{0}^{1, A}(\Omega)$ and $\mathcal{A}_{\lambda}$ is a pseudomonotone operator.

It remains to prove that $\mathcal{A}_{\lambda}$ is coercive for some $\lambda>0$. Using (2.4)

$$
\begin{array}{r}
a \int_{\Omega} \widetilde{E}^{-1}(A(|\nabla T u|))|u| d x=2 a \int_{\{u>\bar{u}\}} \widetilde{E}^{-1}(A(|\nabla \bar{u}|)) \frac{|u|}{2} d x+ \\
2 a \int_{\{u<\underline{u}\}} \widetilde{E}^{-1}(A(|\nabla \underline{u}|)) \frac{|u|}{2} d x+\frac{p_{A}}{2} \int_{\{\underline{u} \leq u \leq \bar{u}\}} \widetilde{E}^{-1}(A(|\nabla u|)) \frac{2 a|u|}{p_{A}} d x \\
\leq 2 a\left[\int_{\{u>\bar{u}\}}\left(A(|\nabla \bar{u}|)+E\left(\frac{|u|}{2}\right)\right) d x+\int_{\{u<\underline{u}\}}\left(A(|\nabla \underline{u}|)+E\left(\frac{|u|}{2}\right)\right) d x\right] \\
+\frac{p_{A}}{2} \int_{\{\underline{u} \leq u \leq \bar{u}\}}\left(A(|\nabla u|)+E\left(\frac{2 a|u|}{p_{A}}\right)\right) d x \leq 2 a \int_{\Omega}(A(|\nabla \bar{u}|)+A(|\nabla \underline{u}|)) d x+ \\
2 a \int_{\Omega} E\left(\frac{|u|}{2}\right) d x+\frac{p_{A}}{2} \int_{\Omega} A(|\nabla u|) d x+\frac{p_{A}}{2} \int_{\underline{u} \leq u \leq \bar{u}} E\left(\frac{2 a \max \{|\underline{u}|,|\bar{u}|\}}{p_{A}}\right) d x .
\end{array}
$$

Since $\underline{u}, \bar{u} \in W^{1, A}(\Omega)$ and $\underline{A} \underset{\epsilon}{\in} \Delta_{2}$ near infinity, (2.17) guarantees that $\int_{\Omega}(A(|\nabla \bar{u}|)+A(|\nabla \underline{u}|)) d x<$ $+\infty$. The assumption $E \ll A_{n}$ allows to use (2.17) that ensures that $\int_{\Omega} E\left(\frac{2 a \max \{\underline{u}, \bar{u}\}}{p_{A}}\right) d x<+\infty$. Thus, there exists a constant $c_{3}>0$ such that

(5.2) $a \int_{\Omega} \widetilde{E}^{-1}(A(|\nabla T u|))|u| d x \leq c_{3}+2 a \int_{\Omega} E\left(\frac{|u|}{2}\right) d x+\frac{p_{A}}{2} \int_{\Omega} A(|\nabla u|) d x$ for all $u \in W_{0}^{1, A}(\Omega)$. 
From hypothesis $(\mathrm{H})$ and $(5.2)$

$$
\begin{aligned}
& \left|\int_{\Omega} f(x, T u, \nabla T u) u d x\right| \leq\left|\int_{\Omega} \sigma(x) u(x) d x\right|+a\left|\int_{\Omega} \widetilde{E}^{-1}(A(|\nabla T u|)) u d x\right| \leq \\
& \quad \leq 2 C\|\sigma\|_{L^{\widetilde{B}}(\Omega)}\|u\|_{W_{0}^{1, A}(\Omega)}+\frac{p_{A}}{2} \int_{\Omega} A(|\nabla u|) d x+2 a \int_{\Omega} E\left(\frac{|u|}{2}\right) d x+c_{3} .
\end{aligned}
$$

From the definition of $p_{A}$

$$
\int_{\Omega} A^{\prime}(|\nabla u|)|\nabla u| d x \geq p_{A} \int_{\Omega} A(|\nabla u|) d x \text { for any } u \in W_{0}^{1, A}(\Omega),
$$

so, choosing $\lambda>a$ and using (4.20), (5.3), (5.4) and (2.15), we obtain

$$
\frac{\left\langle\mathcal{A}_{\lambda}(u), u\right\rangle}{\|u\|_{W_{0}^{1, A}(\Omega)}} \geq \frac{\frac{p_{A}}{2}\|u\|_{W_{0}^{1, A}(\Omega)}^{p_{A}}-2 C\|\sigma\|_{L^{\widetilde{B}}(\Omega)}\|u\|_{W_{0}^{1, A}(\Omega)}+2(\lambda-a) \int_{\Omega} E\left(\frac{|u|}{2}\right) d x-c_{2}-c_{3}}{\|u\|_{W_{0}^{1, A}(\Omega)}}
$$

for all $\|u\|_{W_{0}^{1, A}(\Omega)} \geq 1$, and this leads to

$$
\lim _{\|u\| \rightarrow+\infty} \frac{\left\langle\mathcal{A}_{\lambda}(u), u\right\rangle}{\|u\|_{W_{0}^{1, A}(\Omega)}}=+\infty
$$

Theorem 4.9 guarantees that, there exists $u \in W_{0}^{1, A}(\Omega)$ such that $\mathcal{A}_{\lambda}(u) \equiv 0$. Thus

$$
\int_{\Omega} A^{\prime}(|\nabla u|) \frac{\nabla u}{|\nabla u|} \nabla v d x+\lambda \int_{\Omega} \pi(x, u(x)) v(x) d x-\int_{\Omega} f(x, T u, \nabla T u) v d x=0 .
$$

for all $v \in W_{0}^{1, A}(\Omega)$.

Proof of Theorem 3.6. By Theorem 5.1 there exists a solution $u \in W_{0}^{1, A}(\Omega)$ of the truncated auxiliary problem (5.1) provided $\lambda>0$ is sufficiently large. Let us fix such a $\lambda$ and $u$.

Via comparison arguments we claim that $u \leq \bar{u}$ a.e. in $\Omega$. First of all we observe that it is not restrictive to assume that $E(t)>0$ for $t>0$. Let us use $v=(u-\bar{u})^{+} \in W_{0}^{1, A}(\Omega)$ as test function in (5.5) and (3.3). Then

$$
\int_{\Omega}\left(A^{\prime}(|\nabla u|) \frac{\nabla u}{|\nabla u|}-A^{\prime}(|\nabla \bar{u}|) \frac{\nabla \bar{u}}{|\nabla \bar{u}|}\right) \nabla(u-\bar{u})^{+} d x+\lambda \int_{\Omega} \pi(u(x))(u-\bar{u})^{+} d x \leq 0 .
$$

The convexity of $A$ and (2.5) lead to

$$
\lambda \int_{\{u>\bar{u}\}} E(u-\bar{u}) \leq \lambda \int_{\{u>\bar{u}\}} \widetilde{E}^{-1}(E(u-\bar{u}))(u-\bar{u}) d x \leq 0,
$$

and this, together with $E(t)>0$ for $t>0$, forces $u \leq \bar{u}$ a.e. in $\Omega$. Arguing with $v=(u-\underline{u})^{-} \in$ $W_{0}^{1, A}(\Omega)$ as test function we obtain $u \geq \underline{u}$ a.e. in $\Omega$. Altogether, we can establish for the solution $u$ of the auxiliary truncated problem (5.1) the enclosure property $u \in[\underline{u}, \bar{u}]$. It only remains to confirm that $u \in W_{0}^{1, A}(\Omega)$ is solution of the problem (1.1). Exploiting the inclusion $u \in[\underline{u}, \bar{u}]$, it follows from (4.1) and (4.19) that $T u=u$ and $\Pi(u)=0$. Consequently, since $u$ is a solution of (5.1), then $u \in W_{0}^{1, A}(\Omega)$ becomes a solution of the original problem (1.1). 
Corollary 5.2 Let $\Omega$ be an open set in $\mathbb{R}^{n}$, with $n \geq 2$, such that $|\Omega|<\infty$. Let $A \in C^{1}([0,+\infty))$ be a Young function for which (2.16) holds. Assume also that A satisfies (2.23) and (2.27), or (2.28). Let $\underline{u}$ and $\bar{u}$ be a subsolution and a supersolution of problem (1.1), respectively, with $\underline{u} \leq \bar{u}$ a.e. in $\Omega, \underline{u}, \bar{u} \in W_{0}^{1, A}(\Omega)$, and such that the Carathéodory function $f: \Omega \times \mathbb{R} \times \mathbb{R}^{n} \rightarrow \mathbb{R}$ fulfills

$$
|f(x, s, \xi)| \leq \rho(x)+g(|s|)+a \widetilde{E}^{-1}(A(|\xi|)) \text { a.e. } x \in \Omega, \text { all } s \in[\underline{u}(x), \bar{u}(x)], \xi \in \mathbb{R}^{n},
$$

where $\rho \in L^{\widetilde{A}_{n}}(\Omega)$, a, E, are as in Theorem 3.6 and $g:[0,+\infty[\rightarrow[0,+\infty[$ is a nondecreasing function such that $g(0)=0$ and there exist $s_{0}, k>0$ such that $g(|s|)|s| \leq A_{n}(k|s|)$ for all $|s| \geq s_{0}$. Then problem $(P)$ possesses a nontrivial solution $u \in W_{0}^{1, A}(\Omega)$.

Proof. Put $w:=\max \{|\underline{u}|,|\bar{u}|\}$. We prove that the $g(w) \in L^{\widetilde{A}_{n}}(\Omega)$. Using $(2.5)$

$$
\begin{aligned}
\widetilde{A}_{n}\left(\frac{g(w)}{k}\right) & \leq \widetilde{A}_{n}\left(\frac{g\left(s_{0}\right)}{k}\right)+\widetilde{A}_{n}\left(\frac{A_{n}(k w)}{k|\underline{u}|}\right) \\
& \leq \widetilde{A}_{n}\left(\frac{g\left(s_{0}\right)}{k}\right)+\widetilde{A}_{n}\left(\left(\widetilde{A}_{n}\right)^{-1}\left(A_{n}(k w)\right)\right)=\widetilde{A}_{n}\left(\frac{g\left(s_{0}\right)}{k}\right)+B(k w) .
\end{aligned}
$$

Due to $|\Omega|<\infty$ and (2.29), the function $\widetilde{A}_{n}\left(\frac{g\left(s_{0}\right)}{k}\right)+A_{n}(k w) \in L^{1}(\Omega)$ and this proves that $\sigma(x):=\rho(x)+g(w(x)) \in L^{\widetilde{A}_{n}}(\Omega)$. Thus the conclusion follows from Theorem 3.6.

Corollary 5.3 Let $\Omega$ be an open set in $\mathbb{R}^{n}$, with $n \geq 2$, such that $|\Omega|<\infty$ and $\Omega \in \mathcal{G}\left(\frac{1}{n^{\prime}}\right)$. Let $A \in C^{1}([0,+\infty))$ be a Young function for which (2.16) holds. Assume also that $A$ satisfies (2.23) and (2.27), or (2.28) and that $A_{n} \in \Delta_{2}$ near infinity. Let $\underline{u}$ and $\bar{u}$ be a subsolution and $a$ supersolution of problem (1.1), respectively, with $\underline{u} \leq \bar{u}$ a.e. in $\Omega$, and such that the Carathéodory function $f: \Omega \times \mathbb{R} \times \mathbb{R}^{n} \rightarrow \mathbb{R}$ fulfills (5.6).

Then problem $(P)$ possesses a nontrivial solution $u \in W_{0}^{1, A}(\Omega)$.

Proof. Put $w:=\max \{|\underline{u}|,|\bar{u}|\}$. We prove that the $g(w) \in L^{\widetilde{A}_{n}}(\Omega)$. Unlike the previous corollary now we can't use (2.29), due to the lack of information that sub and supersolutions are in $W_{0}^{1, A}(\Omega)$. However, from $(2.17), A_{n}(k|w|) \in L^{1}(\Omega)$ for all $w \in L^{A_{n}}(\Omega)$ and a fortiori for all $w \in W^{1, A}(\Omega)$, due to (2.24). We can now argue as in Corollary 5.2 to achieve the conclusion.

We are ready for the proof of Theorems 3.2 and 3.3 .

Proof of Theorem 3.2. We construct a subsolution $\underline{u} \leq 0$ a.e., $u \not \equiv 0$, and show that $\bar{u} \equiv 0$ is a supersolution but not a solution to (1.1). Then, we show that $f$ satisfies (5.6).

Put $G_{1}(t)=\int_{0}^{t} g_{1}(\tau) d \tau, t \geq 0$ and consider the functional $J: W_{0}^{1, A}(\Omega) \rightarrow \mathbb{R}$, defined as

$$
J(u)=\int_{\Omega}\left(A(|\nabla u|)+\rho_{1}(x) u-G_{1}(|u|)\right) d x \quad \text { for } u \in W_{0}^{1, A}(\Omega) .
$$

We prove that $J$ is well defined, weakly lower semicontinuous, coercive and that

$$
J^{\prime}(u) v=\int_{\Omega} A^{\prime}(|\nabla u|) \frac{\nabla u}{|\nabla u|} \nabla v d x+\int_{\Omega} \rho_{1}(x) v(x) d x-\int_{\Omega} g_{1}(|u|) \operatorname{sign} u v(x) d x
$$

for all $u, v \in W_{0}^{1, A}(\Omega)$. We examine separately the three integrals.

Due to the convexity of $A$ and Proposition 4.7, the functional $u \mapsto \int_{\Omega} A(|\nabla u|) d x$ is well defined in 
$W_{0}^{1, A}(\Omega)$, convex and of class $C^{1}$.

The embedding $W_{0}^{1, A}(\Omega) \rightarrow L^{A_{n}}(\Omega)$ and (2.8) allow to write

$$
\left|\int_{\Omega} \rho_{1}(x) u d x\right| \leq 2\left\|\rho_{1}\right\|_{L^{\widetilde{A}_{n}(\Omega)}}\|u\|_{L^{A_{n}(\Omega)}} \leq c_{4}\|u\|_{W_{0}^{1, A}(\Omega)} \text { for any } u \in W_{0}^{1, A}(\Omega) .
$$

Thus the functional $\left.u \mapsto \int_{\Omega} \rho_{1}(x)\right) u d x$ is well defined in $W_{0}^{1, A}(\Omega)$, linear and continuous.

Let us consider the functional $u \mapsto \int_{\Omega} G_{1}(|u|) d x, u \in W_{0}^{1, A}(\Omega)$. For any $u \in W_{0}^{1, A}(\Omega)$ and all $x \in \Omega$ one has

$$
G_{1}(|u|) \leq g_{1}(|u|)|u| \leq g_{1}\left(s_{0}\right) s_{0}+A\left(k_{1}|u|\right) .
$$

The assumption $|\Omega|<\infty$ and the $\Delta_{2}$ condition on $A$ (see (2.17)) lead to

$$
g_{1}\left(s_{0}\right) s_{0}+A\left(k_{1}|u|\right) \in L^{1}(\Omega) \text { for all } u \in L^{A}(\Omega)
$$

and a fortiori for all $u \in W_{0}^{1, A}(\Omega)$. This fact will be used often in the proof. First of all it guarantees that the functional $u \mapsto \int_{\Omega} G_{1}(|u|) d x$ is well defined in $W_{0}^{1, A}(\Omega)$. We prove that it is weakly continuous. Let $\left\{u_{k}\right\}$ be a sequence in $W_{0}^{1, A}(\Omega)$ weakly converging to $u \in W_{0}^{1, A}(\Omega)$. Due to the compactness of the embedding $W_{0}^{1, A}(\Omega) \rightarrow L^{A}(\Omega)$ we can find $w \in L^{A}(\Omega)$ such that $\left|u_{k}(x)\right| \leq w(x)$ for a.e. $x \in \Omega$, all $k \in \mathbb{N}$ and, from (5.10), we can apply the Lebesgue theorem to the sequence $\left\{G_{1}\left(\left|u_{k}\right|\right)\right\}$, that is

$$
\lim _{k \rightarrow+\infty} \int_{\Omega} G_{1}\left(\left|u_{k}\right|\right) d x=\int_{\Omega} G_{1}(|u|) d x .
$$

This proves the weak continuity of $u \mapsto \int_{\Omega} G_{1}(|u|) d x$, and the weak lower semicontinuity of $J$. It remains to calculate its derivative. Let $u, v \in W_{0}^{1, A}(\Omega)$ and $\lambda>0, \lambda \ll 1$. Then

$$
\lim _{\lambda \rightarrow 0^{+}} \frac{G_{1}(|u+\lambda v|)-G_{1}(|u|)}{\lambda}=g_{1}(|u|) \operatorname{sign} u v \text { a.e. in } \Omega
$$

and

$$
\left|\frac{G_{1}(|u+\lambda v|)-G_{1}(|u|)}{\lambda}\right| \leq g_{1}(|u|+|v|)|v| \leq g_{1}(|u|+|v|)(|u|+|v|) \leq g_{1}\left(s_{0}\right) s_{0}+A(k(|u|+|v|)) .
$$

From (5.10) we can apply the Lebesgue theorem to $\left\{\frac{G_{1}(|u+\lambda v|)-G_{1}(|u|)}{\lambda}\right\}_{\lambda>0}$, for $\lambda \rightarrow 0^{+}$, and this, together with the regularity properties of the two functionals examined above, leads to (5.8).

To prove the coercivity of $J$ we need the following inequality, that can be found in [BC, Proposition $3.2]$,

$$
\int_{\Omega} A(|u|) d x \leq \int_{\Omega} A\left(\omega_{n}^{-\frac{1}{n}}|\Omega|^{\frac{1}{n}}|\nabla u|\right) d x \text { for all } u \in W_{0}^{1, A}(\Omega) .
$$

Let $\tau:=k_{1} \omega_{n}^{-\frac{1}{n}}|\Omega|^{\frac{1}{n}}$. Note that $\tau<1$.

$$
\begin{gathered}
\int_{\Omega} G_{1}(|u|) d x \leq \int_{\left\{|u| \leq s_{0}\right\}} G_{1}(M) d x+\int_{\left\{|u|>s_{0}\right\}} A\left(k_{1}|u|\right) \\
\leq G_{1}(M)|\Omega|+\int_{\Omega} A(\tau|\nabla u|) d x \leq G_{1}(M)|\Omega|+\tau \int_{\Omega} A(|\nabla u|) d x \text { for all } u \in W_{0}^{1, A}(\Omega) .
\end{gathered}
$$

Take now $u \in W_{0}^{1, A}(\Omega),\|u\|_{W_{0}^{1, A}(\Omega)}>1$ and use (2.15), (5.9) and (5.11)

$$
\frac{J(u)}{\|u\|_{W_{0}^{1, A}(\Omega)}} \geq \frac{(1-\tau) \int_{\Omega} A(|\nabla u|) d x-c_{4}\|u\|_{W_{0}^{1, A}(\Omega)}-G_{1}(M)|\Omega|}{\|u\|_{W_{0}^{1, A}(\Omega)}} \geq p_{A}(1-\tau)\|u\|_{W_{0}^{1, A}(\Omega)}^{p_{A}-1}-c_{4}-\frac{G_{1}(M)|\Omega|}{\|u\|_{W_{0}^{1, A}(\Omega)}} .
$$


This proves that $J$ is coercive. Thus it has a global minimum. Let $\underline{u}$ be a global minimum point for $J$. We prove that $\underline{u} \not \equiv 0$. To this end consider a function $v \in C_{0}^{1}(\Omega), v \leq 0$ and $\rho_{1}(x) v(x) \not \equiv 0$ in $\Omega$. The inequality $\frac{A\left(t_{1}\right)}{A\left(t_{0}\right)}>\left(\frac{t_{1}}{t_{0}}\right)^{p_{A}}$ holds for $t_{1}>t_{0}>0$, then for $t_{1}=|\nabla v|, t_{0}=t|\nabla v|$, we have

$$
J(t v) \leq t^{p_{A}} \int_{\Omega} A(|\nabla v|) d x+t \int_{\Omega} \rho_{1}(x) v d x<0 \text { for } t<1,
$$

and this proves that $J(\underline{u})<0$. Using $J(-|\underline{u}|) \geq J(\underline{u})$ we obtain $\underline{u} \leq 0$ a.e. in $\Omega$.

Now we prove that $\underline{u}$ is a subsolution and $u \equiv 0$ is a supersolution but not a solution to (1.1). Note that

$$
J^{\prime}(\underline{u})(v)=\int_{\Omega} A^{\prime}(|\nabla \underline{u}|) \frac{\nabla \underline{u}}{|\nabla \underline{u}|} \nabla v d x+\int_{\Omega} \rho_{1}(x) v d x+\int_{\Omega} g_{1}(|\underline{u}(x)|) v(x) d x .
$$

Acting with any $v \in W_{0}^{1, A}(\Omega), v \geq 0$, in $J^{\prime}(\underline{u})(v)=0$ and using $(3.1)$

$$
\int_{\Omega} A^{\prime}(|\nabla \underline{u}|) \frac{\nabla \underline{u}}{|\nabla \underline{u}|} \nabla v d x-\int_{\Omega} f(x, \underline{u}, \nabla \underline{u}) v d x \leq 0,
$$

that is $\underline{u}$ is a subsolution to (1.1). Using (3.1) and choosing $v \in W_{0}^{1, A}(\Omega), v \geq 0$

$$
0-\int_{\Omega} f(x, 0,0) v d x \geq \int_{\Omega} \rho_{2}(x) v d x \geq 0,
$$

thus $u \equiv 0$ is a supersolution to (1.1) and the assumptions on $\rho_{2}$ guarantees that it is not a solution. We put $\rho(x)=\max \left\{\rho_{i}(x), i=1,2\right\}, g(|s|)=\max \left\{g_{i}(|s|), i=1,2\right\}$ and use (3.1)

$$
|f(x, s, \xi)| \leq \rho(x)+g(|s|)+a \widetilde{E}^{-1}(A(|\xi|)) \text { for } x \in \Omega, s \in[\underline{u}(x), 0], \quad \xi \in \mathbb{R}^{n} .
$$

Then $f$ satisfies (5.6) and from Corollary 5.2 problem (1.1) has a nontrivial solution $u \in W_{0}^{1, A}(\Omega)$ and $u \in[\underline{u}, 0]$.

From [Ci2, Theorem 2], when $\rho_{1}, \rho_{2} \in L^{\infty}(\Omega)$ then the same holds also for the solution $u$.

Proof of Theorem 3.3. We show that $\underline{u} \equiv 0$ is a subsolution but not a solution to (1.1) and construct a supersolution $\bar{u} \geq 0$ a.e. $u \not \equiv 0$. Then, we show that $f$ satisfies $(5.6)$.

Using (3.2)

$$
0-\int_{\Omega} f(x, 0,0) v d x \leq \int_{\Omega}-\rho_{2}(x) v d x \leq 0 \text { for all } v \in W_{0}^{1, A}(\Omega), v \geq 0 \text { in } \Omega,
$$

thus $\underline{u} \equiv 0$ is a subsolution to (1.1) and the condition on $\rho_{2}$ guarantees that it is not a solution. Put $G_{1}(t)=\int_{0}^{t} g_{1}(\tau) d \tau, t \geq 0$ and consider the functional $J: W_{0}^{1, A}(\Omega) \rightarrow \mathbb{R}$, defined as

$$
J(u)=\int_{\Omega}\left(A(|\nabla u|)-\rho_{1}(x) u-G_{1}(|u|)\right) d x \text { for } u \in W_{0}^{1, A}(\Omega) .
$$

As in Theorem 3.2, we see that $J$ is well defined, weakly lower semicontinuous, coercive and

$$
J^{\prime}(u)(v)=\int_{\Omega} A^{\prime}(|\nabla u|) \frac{\nabla u}{|\nabla u|} \nabla v d x-\int_{\Omega} \rho_{1}(x) v(x) d x-\int_{\Omega} g_{1}(|u(x)|) \operatorname{sign} u(x) v(x) d x .
$$

Let $\bar{u}$ be a global minimum point for $J$. We prove that $\bar{u} \not \equiv 0$. To this end consider a function $v \in C_{0}^{1}(\Omega), v \geq 0, \rho_{2}(x) v(x) \not \equiv 0$ in $\Omega$.

$$
J(t v) \leq t^{p_{A}} \int_{\Omega} A(|\nabla v(x)|) d x-t \int_{\Omega} \rho_{1}(x) v(x) d x<0 \text { for } t<1
$$


and this leads to $J(\bar{u})<0$. Using $J(|\bar{u}|) \geq J(\bar{u})$ we obtain $\bar{u} \geq 0$ a.e. in $\Omega$.

Now we prove that $\bar{u}$ is a supersolution to (1.1). Acting with any $v \in W_{0}^{1, A}(\Omega), v \geq 0$, in $J^{\prime}(\bar{u})(v)=0$ and using the inequality on the right in (3.2)

$$
\int_{\Omega} A^{\prime}(|\nabla \bar{u}|) \frac{\nabla \bar{u}}{|\nabla \bar{u}|} \nabla v d x-\int_{\Omega} f(x, \bar{u}, \nabla \bar{u}) v d x \geq 0
$$

Arguing as in Theorem 3.2 we see that $f$ satisfies (5.6), and problem (1.1) has a nontrivial solution $u \in[0, \bar{u}]$.

\section{References}

[Ad] R.A.Adams, "Sobolev Spaces", Academic Press, New York, 1975.

[BC] G.Barletta, A.Cianchi, Dirichlet problems for fully anisotropic elliptic equations. Proc. Roy. Soc. Edinburgh Sect. A 147 (2017), no. 1, 25-60.

[CLM] S.Carl, V.K.Le, D.Motreanu, "Nonsmooth variational problems and their inequalities. Comparison principles and applications", Springer Monographs in Mathematics. Springer, New York, 2007.

[Ci1] A.Cianchi, A sharp embedding theorem for Orlicz-Sobolev spaces, Indiana Univ. Math. J. 45 (1996), 39-65.

[Ci2] A.Cianchi, Boundedness of solutions to variational problems under general growth conditions, Comm. Part. Diff. Eq. 22 (1997), 1629-1646.

[Ci3] A.Cianchi, A fully anisotropic Sobolev inequality, Pacific J. Math. 196 (2000), 283-295.

[CM] M.Chmara, J.Maksymiuk, Mountain pass type periodic solutions for Euler-Lagrange equations in anisotropic Orlicz-Sobolev space, J. Math. Anal. Appl. 470 (2019), no. 1, 584ï£j598.

[FGM] D. De Figueiredo, M. Girardi, M. Matzeu, Semilinear elliptic equations with dependence on the gradient via mountain-pass techniques, Differ. Integral Equ. 17(1-2) (2004), 119-126.

[DF] G.Dong, X.Fang, Differential equations of divergence form in separable Musielak-OrliczSobolev spaces, Bound. Value Probl. (2016), Paper No. 106, 19 pp.

[FMP] F.Faraci, D.Motreanu, D.Puglisi, Positive solutions of quasi-linear elliptic equations with dependence on the gradient, Calc. Var. Partial Differential Equations 54 (2015), no. 1, 525538.

[FMMT] L.F.O.Faria, O.H.Miyagaki, D.Motreanu, M.Tanaka, Existence results for nonlinear elliptic equations with Leray-Lions operator and dependence on the gradient, Nonlinear Anal. 96 (2014), 154ї¡166.

[GW] L.Gasiński, P.Winkert, Existence and uniqueness results for double phase problems with convection term, J. Differential Equations, https://doi.org/10.1016/j.jde.2019.10.022

[G] N.Grenon, Existence and Comparison Results for Quasilinear Elliptic Equations with Critical Growth in the Gradient, J. Differential Equations 171 (2001), no. 1, 1-23. 
[LI] P.Hajlasz, Z.Liu, Zhuomin, A compact embedding of a Sobolev space is equivalent to an embedding into a better space, Proc. Amer. Math. Soc. 138 (2010), no. 9, 3257-3266.

[HH] P.Harjulehto, P.Hästö, "Orlicz spaces and generalized Orlicz spaces. Lecture Notes in Mathematics, 2236, Springer, Cham, 2019. x+167 pp. ISBN: 978-3-030-15099-0; 978-3-030-15100-3.

[H] J.Heinonen, T.Kilpelï¡inen, O.Martio, "Nonlinear potential theory of degenerate elliptic equations, Oxford Mathematical Monographs. Oxford Science Publications. The Clarendon Press, Oxford University Press, New York, 1993.

[KrRu] M.A.Krasnosel'skii, Ja.B.Rutickii, "Convex functions and Orlicz spaces, Groningen: Noordhoff, 1961.

[M] V.Maz’ya, "Sobolev Spaces", Springer-Verlag, Berlin, 1985.

[MMM] D.Motreanu, V.V.Motreanu, A.Moussaoui, Location of nodal solutions for quasilinear elliptic equations with gradient dependence, Discrete Contin. Dyn. Syst. Ser. S 11 (2018), no. 2, 293-307.

[MT] D.Motreanu, E.Tornatore, Location of solutions for quasi-linear elliptic equations with general gradient dependence. Electron, J. Qual. Theory Differ. Equ. 2017, Paper No. 87, 10 pp.

[MW] D.Motreanu, P.Winkert, Existence and asymptotic properties for quasilinear elliptic equations with gradient dependence, Appl. Math. Lett. 95 (2019), 78-84.

[RR1] M.M.Rao \& Z.D.Ren, "Theory of Orlicz spaces", Marcel Dekker, New York, 1991.

[RR2] M.M.Rao \& Z.D.Ren, "Applications of Orlicz spaces", Marcel Dekker, New York, 2002.

[R] D.Ruiz, A priori estimates and existence of positive solutions for strongly nonlinear problems, J. Differential Equations 199 (2004), no. 1, 96-114.

[Sch] G. Schappacher, A notion of Orlicz spaces for vector valued functions, Appl. Math. 50 (2005), $355-386$.

[T] M.Tanaka, Existence of a positive solution for quasilinear elliptic equations with nonlinearity including the gradient, Bound. Value Probl. 2013, 2013:173, 11 pp. 